JOURNAL OF THE AMERICAN MATHEMATICAL SOCIETY

Volume 21, Number 4, October 2008, Pages 925-950

S 0894-0347(07)00582-6

Article electronically published on October 10, 2007

\title{
HARDY-LIEB-THIRRING INEQUALITIES FOR FRACTIONAL SCHRÖDINGER OPERATORS
}

\author{
RUPERT L. FRANK, ELLIOTT H. LIEB, AND ROBERT SEIRINGER
}

\section{INTRODUCTION}

We shall generalize several well known inequalities about the negative spectrum of Schrödinger-like operators on $\mathbb{R}^{d}$. As an application of our results we shall give a proof of the 'stability of relativistic matter' - one which goes further than previous proofs by permitting the inclusion of magnetic fields for values of the nuclear charge all the way up to $Z \alpha=2 / \pi$, which is the critical value in the absence of a field.

There are three main inequalities to which we refer. The first is Hardy's inequality, whose classical form for $d \geq 3$ is the following. (In this introduction we shall not be precise about the space of functions in question, but will be precise later on.)

$$
\int_{\mathbb{R}^{d}}\left(|\nabla u(x)|^{2}-\frac{(d-2)^{2}}{4|x|^{2}}|u(x)|^{2}\right) d x \geq 0 .
$$

The second is Sobolev's inequality for $d \geq 3$,

$$
\int_{\mathbb{R}^{d}}|\nabla u(x)|^{2} d x \geq S_{2, d}\left\{\int_{\mathbb{R}^{d}}|u(x)|^{2 d /(d-2)} d x\right\}^{1-2 / d}=S_{2, d}\|u\|_{2 d /(d-2)}^{2} .
$$

The third is the Lieb-Thirring (LT) inequality [LTh] for the Schrödinger operator $H=-\Delta-V(x)$. If its negative eigenvalues are denoted by $-\lambda_{1} \leq-\lambda_{2} \leq \cdots$, and if $\gamma \geq 0$, then

$$
\sum_{j} \lambda_{j}^{\gamma} \leq L_{\gamma, d} \int_{\mathbb{R}^{d}} V(x)_{+}^{\gamma+d / 2} d x=L_{\gamma, d}\left\|V_{+}\right\|_{\gamma+d / 2}^{\gamma+d / 2} .
$$

This holds if and only if $\gamma \geq \frac{1}{2}$ when $d=1, \gamma>0$ when $d=2$ and $\gamma \geq 0$ when $d \geq 3$. (Here and in the sequel $t_{-}:=\max \{0,-t\}$ and $t_{+}:=\max \{0, t\}$ denote the negative and positive parts of $t$.)

By duality, (1.2) is equivalent to the fact that the Schrödinger operator $H=$ $-\Delta-V$ does not have negative eigenvalues if $\left\|V_{+}\right\|_{d / 2} \leq S_{2, d}$. On the other hand, (1.3) gives an upper bound to the number of negative eigenvalues in terms of $\left\|V_{+}\right\|_{d / 2}$ when $\gamma=0$ and it estimates the magnitude of these eigenvalues when $\gamma>0$.

All three inequalities can be generalized by the inclusion of a magnetic vector potential $A$ (related to the magnetic field $B$ by $B=\operatorname{curl} A$ ). That is, $\nabla$ is replaced

Received by the editors October 18, 2006.

2000 Mathematics Subject Classification. Primary 35P15; Secondary 81Q10.

Key words and phrases. Hardy inequality, relativistic Schrödinger operator, Lieb-Thirring inequalities, Sobolev inequalities, stability of matter, diamagnetic inequality. 
by $\nabla-i A(x)$, and $\Delta$ by $(\nabla-i A(x))^{2}$. When $A$ is included the inequalities (1.1) and (1.2) remain valid with the same constants $(d-2)^{2} / 4$ and $S_{2, d}$, respectively. All known proofs of (1.3) remain true with the inclusion of $A$ and yield a constant $L_{\gamma, d}$ that is independent of $A$, but it is not known if the unknown sharp constant in (1.3) is independent of $A$. The inclusion of $A$ is easily done in (1.1), (1.2) by using the diamagnetic inequality, but the inclusion in (1.3) is more delicate; one uses the Feynman-Kac path integral formula to show that for each $x, y \in \mathbb{R}^{d}$ and $t, \tau>0$, the $A$-field reduces the magnitude of the heat kernel $e^{t(\nabla-i A)^{2}}(x, y)$ relative to $e^{t \Delta}(x, y)$, and hence reduces the resolvent kernel $\left|\left[-(\nabla-i A)^{2}+\tau\right]^{-1}(x, y)\right|$ relative to $[-\Delta+\tau]^{-1}(x, y)$.

From $\mathrm{BVa}$, one can deduce that (1.1) and (1.2) can be combined as follows: For $\varepsilon>0$,

$$
\int_{\mathbb{R}^{d}}\left(|\nabla u(x)|^{2}-\frac{(d-2)^{2}}{4|x|^{2}}|u(x)|^{2}+|u(x)|^{2}\right) d x \geq S_{2, d, \varepsilon}^{\prime}\|u\|_{2 d /(d-2+\varepsilon)}^{2}
$$

with $S_{2, d, \varepsilon}^{\prime} \rightarrow 0$ as $\varepsilon \rightarrow 0$. Note the extra term $|u(x)|^{2}$ on the left side to account for the fact that the left and right sides behave differently under scaling; examples show that it really is necessary to have $\varepsilon>0$ here.

In [EkFr], a parallel extension of (1.3) for the negative eigenvalues $-\lambda_{j}$ of the Schrödinger operator $H=-\Delta-(d-2)^{2} /\left(4|x|^{2}\right)-V$ is proved. For $\gamma>0$ and $d \geq 3$,

$$
\sum_{j} \lambda_{j}^{\gamma} \leq L_{\gamma, d}^{\prime} \int_{\mathbb{R}^{d}} V(x)_{+}^{\gamma+d / 2} d x=L_{\gamma, d}^{\prime}\left\|V_{+}\right\|_{\gamma+d / 2}^{\gamma+d / 2},
$$

with $L_{\gamma, d}^{\prime} \geq L_{\gamma, d}$. Note that there is no need for an $\varepsilon$ in (1.5); the fact that $\varepsilon \neq 0$ in (1.4) is reflected here in the fact that $\gamma>0$ is needed. As before, a magnetic vector potential can easily be included in (1.4), but it does not seem easy to include a magnetic field in (1.5) by the methods in $[\mathrm{EkFr}$.

Our goal here is to extend these results in several ways. One extension is to include a magnetic field in (1.5). Another is to consider fractional powers of the (magnetic) Laplacian, i.e., to the case in which $|\nabla-i A|^{2}$ is replaced by $|\nabla-i A|^{2 s}$ with $0<s<\min \{1, d / 2\}$ (which means that we can now include one- and twodimensions). This is a significant generalization because the operator $(-\Delta)^{s}$ is not a differential operator and it is not 'local'. Really different techniques will be needed. In particular, we shall use the heat kernel to prove the analog of (1.5), in the manner of [L1]. A bound on this kernel, in turn, will be derived from a Sobolevlike inequality (the analogue of (1.4) ) by using an analogue of Nash's inequality, as explained in [LLo]. The appropriate inequalities are naturally formulated in a weighted space with measure $|x|^{-\beta} d x$ for $\beta>0$. Therefore, a pointwise bound on the heat kernel for a weighted 'Hardy' operator

$$
\exp \left\{-t|x|^{\alpha}\left((-\Delta)^{s}-\mathcal{C}_{s, d}|x|^{-2 s}+1\right)|x|^{\alpha}\right\}
$$

for appropriate $\alpha>0$ will be needed and will not be straightforward to obtain.

In the dimension most relevant for physics, $d=3$, the earlier case $s=1$ may be called the non-relativistic case, while the new result for $s=1 / 2$ may be called the relativistic case. Indeed, the resulting LT inequality, together with some of the methodology in [LY], yields a new proof of the stability of relativistic matter, which will be sketched in Subsection 2.2. The main point, however, is that this new proof allows for an arbitrary magnetic vector potential $A$. Since the constant in 
the relativistic $(s=1 / 2)$ Hardy inequality that replaces $(d-2)^{2} / 4$ is $2 / \pi$ (which is the same as the critical value of $Z \alpha$ in the field-free relativistic case), we conclude that we can simultaneously have an arbitrary $A$-field and the critical value of $Z \alpha$, the nuclear charge times the fine-structure constant. See Theorem 2.7. Up to now it was not possible to have both an arbitrary field and $Z \alpha=2 / \pi$. Therefore, the proof of the analogue of (1.5) for $s=1 / 2$ with an $A$-field opens a slightly improved perspective on the interaction of matter and radiation.

\section{MAIN RESULTS}

2.1. Hardy-Lieb-Thirring inequalities. We recall the Hardy-type inequality

$$
\int_{\mathbb{R}^{d}}|x|^{-2 s}|u(x)|^{2} d x \leq \mathcal{C}_{s, d}^{-1} \int_{\mathbb{R}^{d}}|\xi|^{2 s}|\hat{u}(\xi)|^{2} d \xi, \quad u \in C_{0}^{\infty}\left(\mathbb{R}^{d}\right),
$$

valid for $0<2 s<d$. Here

$$
\hat{u}(\xi):=(2 \pi)^{-d / 2} \int_{\mathbb{R}^{d}} u(x) e^{-i \xi \cdot x} d x, \quad \xi \in \mathbb{R}^{d},
$$

denotes the Fourier transform of $u$. The sharp constant in (2.1),

$$
\mathcal{C}_{s, d}:=2^{2 s} \frac{\Gamma^{2}((d+2 s) / 4)}{\Gamma^{2}((d-2 s) / 4)}
$$

was found by Herbst [He] and then by Beckner Be1] and Yafaev Y Ya. Moreover, this constant is not achieved in the class of functions for which both sides are finite L2, Be1, Ya. In the case $0<s<\min \{1, d / 2\}$, this fact can also be deduced from our ground state representation in Proposition 4.1, which therefore represents an independent proof of (2.1). See Remark 4.2 below.

We denote $D=-i \nabla$. Consider a magnetic vector potential $A \in L_{\text {loc }}^{2}\left(\mathbb{R}^{d}, \mathbb{R}^{d}\right)$ and the self-adjoint operator $(D-A)^{2}$ in $L^{2}\left(\mathbb{R}^{d}\right)$. For $0<s \leq 1$ we define the operator $|D-A|^{2 s}:=\left((D-A)^{2}\right)^{s}$ by the spectral theorem. One form of the diamagnetic inequality states that if $0<s \leq 1$ and $u \in \operatorname{dom}|D-A|^{s}$, then $|u| \in H^{s}\left(\mathbb{R}^{d}\right)$ and

$$
\left\|(-\Delta)^{s / 2}|u|\right\|^{2} \leq\left\||D-A|^{s} u\right\|^{2} \text {. }
$$

Here and in the sequel, $\|\cdot\|=\|\cdot\|_{2}$ denotes the $L^{2}$-norm. We refer to Remark 6.2 for more details concerning (2.3). Combining this inequality with the Hardy inequality (2.1) we find that the quadratic form

$$
h_{s, A}[u]:=\left\||D-A|^{s} u\right\|^{2}-\mathcal{C}_{s, d}\left\||x|^{-s} u\right\|^{2}
$$

is non-negative on $\operatorname{dom}|D-A|^{s}$ if $0<s<\min \{1, d / 2\}$. We use the same notation for its closure and denote by

$$
H_{s, A}=|D-A|^{2 s}-\mathcal{C}_{s, d}|x|^{-2 s}
$$

the corresponding self-adjoint operator in $L^{2}\left(\mathbb{R}^{d}\right)$.

Our main result is

Theorem 2.1 (Hardy-Lieb-Thirring inequalities). Let $\gamma>0$ and $0<s<\min \{1, d / 2\}$. Then there exists a constant $L_{\gamma, d, s}>0$ such that for all $V$ and $A$

$$
\operatorname{tr}\left(|D-A|^{2 s}-\mathcal{C}_{s, d}|x|^{-2 s}-V\right)_{-}^{\gamma} \leq L_{\gamma, d, s} \int_{\mathbb{R}^{d}} V(x)_{+}^{\gamma+d / 2 s} d x .
$$


Remark 2.2. For $d \geq 3$, Theorem 2.1 also holds for $s=1$. In the non-magnetic case $A=0$, this has been proved in $[\mathrm{EkFr}$. In the present paper, we present an independent proof of this result, which allows for the inclusion of an $A$-field. For simplicity, we restrict our attention to $s<1$ in the following, and comment on the (simpler) case $s=1$ in Subsection 6.4.

Theorem 2.1 will be proved in Section 5. The main ingredient in its proof is a Sobolev-type inequality which might be of independent interest and which we shall present in the remainder of this subsection.

For the case $A=0$ we shall drop the index $A$ from the notation, i.e.,

$$
h_{s}[u]=\int_{\mathbb{R}^{d}}|\xi|^{2 s}|\hat{u}(\xi)|^{2} d \xi-\mathcal{C}_{s, d} \int_{\mathbb{R}^{d}}|x|^{-2 s}|u(x)|^{2} d x .
$$

We denote the closure of this form by the same letter. In particular, its domain $\operatorname{dom} h_{s}$ is the closure of $C_{0}^{\infty}\left(\mathbb{R}^{d}\right)$ with respect to the norm $\left(h_{s}[u]+\|u\|^{2}\right)^{1 / 2}$. Note that $H^{s}\left(\mathbb{R}^{d}\right) \subset \operatorname{dom} h_{s}$ with strict inclusion. In particular, there exist functions $u \in \operatorname{dom} h_{s}$ for which both sides of (2.1) are infinite.

Hardy's inequality (2.1) implies that $h_{s}$ is non-negative. The following theorem shows that for functions of compact support it even satisfies a Sobolev-type inequality; i.e., $h_{s}[u]$ can be bounded from below by an $L^{q}$-norm of $u$.

Theorem 2.3 (Local Sobolev-Hardy inequality). Let $0<s<\min \{1, d / 2\}$ and $1 \leq q<2^{*}=2 d /(d-2 s)$. Then there exists a constant $C_{q, d, s}>0$ such that for any domain $\Omega \subset \mathbb{R}^{d}$ with finite measure $|\Omega|$ one has

$$
\|u\|_{q}^{2} \leq C_{q, d, s}|\Omega|^{2\left(\frac{1}{q}-\frac{1}{2^{*}}\right)} h_{s}[u], \quad u \in C_{0}^{\infty}(\Omega) .
$$

Remark 2.4. Note that the exponent $q$ is strictly smaller than the critical Sobolev exponent $2^{*}=2 d /(d-2 s)$. By considering functions which diverge like $|x|^{-(d-2 s) / 2}$ at $x=0$, it is easy to see that the inequality (2.6) cannot hold if $q$ is replaced by $2^{*}$.

We note that the analogue of Theorem 2.3 in the local case $s=1$ is proved in BVa. Theorem 2.3 will be proved in Section 3. where we also deduce the following corollary from it.

Corollary 2.5 (Global Sobolev-Hardy inequality). Let $0<s<\min \{1, d / 2\}$ and $2 \leq q<2^{*}=2 d /(d-2 s)$. Then there exists a constant $C_{q, d, s}^{\prime}>0$ such that

$$
\|u\|_{q}^{2} \leq C_{q, d, s}^{\prime}\left(h_{s}[u]+\|u\|^{2}\right), \quad u \in C_{0}^{\infty}\left(\mathbb{R}^{d}\right) .
$$

Note that (2.7) may be written in the scale-invariant form

$$
\|u\|_{q} \leq C_{q, d, s}^{\prime \prime} h_{s}[u]^{\frac{d}{2 s}\left(\frac{1}{2}-\frac{1}{q}\right)}\|u\|^{\frac{d}{s}\left(\frac{1}{q}-\frac{1}{2^{*}}\right)},
$$

where $C_{q, d, s}^{\prime \prime}$ can be expressed explicitly in terms of $C_{q, d, s}^{\prime}$. This inequality follows from applying (2.7) to functions of the form $u_{\lambda}(x)=u(\lambda x)$ and then optimizing over the choice of $\lambda$.

Although the sharp constants in Theorems 2.1 and 2.3, as well as in Corollary 2.5. are unknown, explicit upper bounds involving a certain variational expression can be deduced from our proof. In Appendix A, we do evaluate explicit bounds on the constants in Theorem 2.3 in the special case $d=3, s=1 / 2$, which is the most interesting case from a physical point of view, as will be explained in the next subsection. 
Remark 2.6. Corollary 2.5 is one of the main ingredients in our proof of Theorem 2.1. On the other hand, Corollary 2.5 is an easy consequence of Theorem 2.1. except for the value of the constants. In fact, (2.5) implies that

$$
h_{s}[u] \geq \int_{\mathbb{R}^{d}} V(x)|u(x)|^{2} d x-\left(L_{\gamma, d, s} \int_{\mathbb{R}^{d}} V(x)_{+}^{\gamma+d / 2 s} d x\right)^{1 / \gamma}\|u\|^{2}
$$

for all $u \in \operatorname{dom} h_{s}$ and all $V \in L^{\gamma+d / 2 s}\left(\mathbb{R}^{d}\right)$. Equation (2.8) follows by optimizing the right side over all $V$.

2.2. Stability of relativistic matter. We shall now explain how the inequalities in Theorem 2.1 can be used to prove stability of relativistic matter in the presence of an external magnetic field. The proof works up to and including the critical value $Z \alpha=2 / \pi$, which is a new result and solves a problem that has been open for a long time. The allowed values of $\alpha$ are discussed after Theorem 2.7. We refer to [L3, L4] for a review of this topic.

We consider $N$ electrons of mass $m \geq 0$ with $q$ spin states $(q=2$ for real electrons) and $K$ fixed nuclei with (distinct) coordinates $R_{1}, \ldots, R_{K} \in \mathbb{R}^{3}$ and charges $Z_{1}, \ldots, Z_{K}>0$. A pseudo-relativistic description of the corresponding quantum-mechanical system is given by the Hamiltonian

$$
H_{N, K}=\sum_{j=1}^{N}\left(\sqrt{\left(D_{j}-\sqrt{\alpha} A\left(x_{j}\right)\right)^{2}+m^{2}}-m\right)+\alpha V_{N, K}\left(x_{1}, \ldots, x_{N}\right) .
$$

The Pauli exclusion principle dictates that $H_{N, K}$ acts on functions in the antisymmetric $N$-fold tensor product of $L^{2}\left(\mathbb{R}^{3} ; \mathbb{C}^{q}\right)$. Here we use units where $\hbar=c=1$, $\alpha>0$ is the fine structure constant, and

$$
\begin{aligned}
V_{N, K}\left(x_{1}, \ldots, x_{N}\right):= & \sum_{1 \leq i<j \leq N}\left|x_{i}-x_{j}\right|^{-1}-\sum_{j=1}^{N} \sum_{k=1}^{K} Z_{k}\left|x_{j}-R_{k}\right|^{-1} \\
& +\sum_{1 \leq k<l \leq K} Z_{k} Z_{l}\left|R_{k}-R_{l}\right|^{-1} .
\end{aligned}
$$

Stability of matter means that $H_{N, K}$ is bounded from below by a constant times $(N+K)$, independently of the positions $R_{k}$ of the nuclei.

By combining the methods in $[\mathrm{LY}]$ and our Theorem [2.1, one can prove the following

Theorem 2.7 (Stability of relativistic matter). There is an $\alpha_{\mathrm{c}}>0$ such that for all $N, K, q \alpha \leq \alpha_{\mathrm{c}}$ and $\alpha \max \left\{Z_{1}, \ldots, Z_{K}\right\} \leq 2 / \pi$ one has

$$
H_{N, K} \geq-m N \text {. }
$$

The constant $\alpha_{\mathrm{c}}$ can be chosen independently of $m, A$ and $R_{1}, \ldots, R_{K}$.

The constant $\alpha_{\mathrm{c}}$ in Theorem 2.7 depends on the optimal constant in the HardyLT inequality (2.5) for $d=3$ and $s=1 / 2$. A bound on this constant, in turn, can be obtained from our proof in terms of the constant in the Sobolev-Hardy inequalities (2.6). We do derive a bound on the relevant constants in Appendix A, but these bounds are probably far from optimal. In particular, the available constants do not yield realistic values of $\alpha_{\mathrm{c}}$ so far.

After completing this work, we discovered a different proof of the special case of the Hardy-LT inequality needed in the proof of Theorem 2.7. namely the case 
where the potential $V$ is constant inside the unit ball, and infinite outside. In this special case a substantially improved constant can be obtained, and this permits the conclusion that Theorem 2.7 holds for the physical value of $\alpha$, which equals $\alpha \approx 1 / 137$. We refer to FLS for details.

We briefly outline the proof of Theorem 2.7. An examination of the proof in [LY] shows that there are two places that do not permit the inclusion of a magnetic vector potential $A$. These are Theorem 9 (Localization of kinetic energy - general form) and Theorem 11 (Lower bound to the short-range energy in a ball). Our Lemma B.1 in Appendix B is precisely the extension of Theorem 9 to the magnetic case. This lemma implies that Theorem 10 in $[\mathrm{LY}]$ also holds in the magnetic case, without change except for replacing $|D|$ by $|D-A|$.

Our Theorem 2.1 can be used instead of Theorem 11 in [LY]. In fact, the left side of (3.20) in [LY] is bounded from below by $q\|\chi\|_{\infty}^{2}$ times the sum of the negative eigenvalues of $|D-A|-\frac{2}{\pi}|x|^{-1}-C R^{-1} \theta_{R}(x)$, where $\theta_{R}$ denotes the characteristic function of a ball of radius $R$. By Theorem 2.1, this latter sum is bounded from below by a constant times $C^{4} R^{-1}$. The resulting bound is of the same form as the right side of (3.20), except for the constant. It is this constant that determines the maximally allowed value of the fine structure constant, $\alpha_{\mathrm{c}}$. The rest of the proof remains unchanged. Note that, in particular, the Daubechies inequality Dau remains true also in the presence of a magnetic field.

2.3. Outline of the paper. Before giving the proofs of our main results, we pause to outline the structure of this paper.

- In Section 3, we give the proof of the Sobolev-Hardy inequalities in Theorem 2.3 and Corollary 2.5 .

- In Section 4 we prove what is customarily called the "ground state representation" in Proposition 4.1, except that here the "ground state" fails to be an $L^{2}$ function. Such a representation for fractional differential operators does not seem to have appeared in the literature before.

- In Section 5 we give the proof of our main Theorem 2.1 about Hardy-LT inequalities. We first consider the non-magnetic case $A=0$. One of the key ingredients is the ground state representation obtained in Section 4. which allows us to prove a certain contraction property of the heat kernel in some weighted $L^{1}$-spaces. Nash's argument [LLo, Sect. 8.15-18] then allows us to translate the Sobolev-Hardy inequalities in Corollary 2.5 into pointwise bounds on the heat kernel in an appropriate weighted $L^{p}$-space. These bounds lead to the Hardy-LT inequalities via the trace formula in Proposition 5.3 in the spirit of [L1].

- Finally, in Section 6 we derive diamagnetic inequalities which will allow us to extend the proof of Theorem 2.1 to the magnetic case.

\section{Sobolev-Hardy inequalities}

Our goal in this section is to prove Theorem 2.3 and Corollary 2.5. We start with a short outline of the structure of the proof.

Our proof is based on the fact that we can control the singularity of $H_{s} \psi$ near the origin if we know the singularity of $\psi$ at that point (cf. Lemma 3.3). Theorem 2.3 follows by observing that the $L^{q}$-norm of a symmetric decreasing function can be bounded above by integrating the function against $|x|^{d(1 / q-1)}$; see Lemma 3.4. 
Moreover, it is enough to restrict one's attention to symmetric decreasing functions. Corollary 2.5 follows from Theorem 2.3 by an IMS-type localization argument; see Lemma 3.5

We present some auxiliary results in Subsection 3.1. Subsections 3.2 and 3.3 contain the proofs of Theorem 2.3 and Corollary 2.5, respectively.

3.1. Auxiliary material. We start with the following integral representation of the operator $(-\Delta)^{s}$.

Lemma 3.1. Let $d \geq 1$ and $0<s<1$. Then for all $u \in H^{s}\left(\mathbb{R}^{d}\right)$

$$
\int_{\mathbb{R}^{d}}|\xi|^{2 s}|\hat{u}(\xi)|^{2} d \xi=a_{s, d} \int_{\mathbb{R}^{d}} \int_{\mathbb{R}^{d}} \frac{|u(x)-u(y)|^{2}}{|x-y|^{d+2 s}} d x d y
$$

where

$$
a_{s, d}:=2^{2 s-1} \pi^{-d / 2} \frac{\Gamma((d+2 s) / 2)}{|\Gamma(-s)|} .
$$

Lemma 3.1 is well known; we sketch the proof for the sake of completeness.

Proof. For fixed $y$ we change coordinates $z=x-y$ and apply Plancherel. Recalling that $(u(\cdot+z))^{\wedge}(\xi)=e^{i \xi \cdot z} \hat{u}(\xi)$ we obtain

$$
\iint \frac{|u(x)-u(y)|^{2}}{|x-y|^{d+2 s}} d x d y=\int\left(\int|z|^{-d-2 s}\left|e^{i \xi \cdot z}-1\right|^{2} d z\right)|\hat{u}(\xi)|^{2} d \xi .
$$

The integral in brackets is of the form $c_{s, d}|\xi|^{2 s}$, with

$$
\begin{aligned}
c_{s, d} & :=\int_{0}^{\infty} \int_{\mathbb{S}^{d-1}}\left|e^{i r \omega \cdot \theta}-1\right|^{2} d \theta r^{-2 s-1} d r \\
& =2 \int_{0}^{\infty}\left(\left|\mathbb{S}^{d-1}\right|-(2 \pi)^{d / 2} r^{-(d-2) / 2} J_{(d-2) / 2}(r)\right) r^{-2 s-1} d r .
\end{aligned}
$$

Here, $J_{(d-2) / 2}$ is the Bessel function of the first kind of order $(d-2) / 2$ AbSt]. Recall that $\left|\mathbb{S}^{d-1}\right|=2 \pi^{d / 2} / \Gamma(d / 2)$. The formula (3.2) for $c_{s, d}=a_{s, d}^{-1}$ now follows from

$$
\int_{0}^{\infty} r^{-z}\left(J_{(d-2) / 2}(r)-2^{-(d-2) / 2} \Gamma(d / 2)^{-1} r^{(d-2) / 2}\right) d r=2^{-z} \frac{\Gamma((d-2 z) / 4)}{\Gamma((d+2 z) / 4)}
$$

for $d / 2<\operatorname{Re} z<(d+4) / 2$; see [Ya, $(2.20)]$.

Let us recall that $|x|^{-\alpha}$ is a tempered distribution for $0<\alpha<d$ with Fourier transform

$$
b_{\alpha}\left(|\cdot|^{-\alpha}\right)^{\wedge}(\xi)=b_{d-\alpha}|\xi|^{-d+\alpha}, \quad b_{\alpha}:=2^{\alpha / 2} \Gamma(\alpha / 2)
$$

(see, e.g., [LLO, Thm. 5.9], where another convention for the Fourier transform is used, however). We now assume that $s<d / 2$. Then $(-\Delta)^{s}|x|^{-\alpha}$ is an $L_{\mathrm{loc}}^{1}$-function for $0<\alpha<d-2 s$ and

$$
\left((-\Delta)^{s}-\mathcal{C}_{s, d}|x|^{-2 s}\right)|x|^{-\alpha}=\Phi_{s, d}(\alpha)|x|^{-\alpha-2 s},
$$

where $\mathcal{C}_{s, d}$ is defined in (2.2) and

$$
\begin{aligned}
\Phi_{s, d}(\alpha) & :=\frac{b_{\alpha+2 s} b_{d-\alpha}}{b_{d-\alpha-2 s} b_{\alpha}}-\mathcal{C}_{s, d} \\
& =2^{2 s}\left(\frac{\Gamma((\alpha+2 s) / 2) \Gamma((d-\alpha) / 2)}{\Gamma((d-\alpha-2 s) / 2) \Gamma(\alpha / 2)}-\frac{\Gamma^{2}((d+2 s) / 4)}{\Gamma^{2}((d-2 s) / 4)}\right) .
\end{aligned}
$$


Later on we will need the following information about the $\alpha$-dependence of $\Phi_{s, d}$.

Lemma 3.2. The function $\Phi_{s, d}$ is negative and strictly increasing in $(0,(d-2 s) / 2)$ with $\Phi_{s, d}((d-2 s) / 2)=0$.

Proof. First one checks that

$$
\lim _{\alpha \rightarrow 0} \Phi_{s, d}(\alpha)=-\mathcal{C}_{s, d}<0, \quad \Phi_{s, d}((d-2 s) / 2)=0 .
$$

Now we abbreviate $\beta:=\alpha / 2, r:=d / 2$ and write

$$
f(\beta):=\Gamma(\beta) / \Gamma(r-\beta), \quad g(\beta):=f(\beta+s) / f(\beta),
$$

so that $\Phi_{s, d}(\alpha)=2^{2 s} g(\beta)-\mathcal{C}_{s, d}$. In view of (3.6) it suffices to verify that $g(\beta)$ is strictly increasing with respect to $\beta \in(0,(r-s) / 2)$. One finds that

$$
\frac{f^{\prime}(\beta)}{f(\beta)}=\frac{\Gamma^{\prime}(\beta)}{\Gamma(\beta)}+\frac{\Gamma^{\prime}(r-\beta)}{\Gamma(r-\beta)}=\psi(\beta)+\psi(r-\beta)
$$

with $\psi=\Gamma^{\prime} / \Gamma$ the Digamma function. Hence

$$
g^{\prime}(\beta)=g(\beta)\left(\frac{f^{\prime}(\beta+s)}{f(\beta+s)}-\frac{f^{\prime}(\beta)}{f(\beta)}\right)=g(\beta) \int_{\beta}^{\beta+s} h(t) d t
$$

where, in view of (3.7) $, h(t):=\psi^{\prime}(t)-\psi^{\prime}(r-t)$. Since $\psi^{\prime}$ is strictly decreasing (see AbSt, (6.4.1)]), one has $h(t)>0$ for $t \in(0, r / 2)$. This proves that $g^{\prime}(\beta)>0$ for all $\beta \in(0,(r-2 s) / 2)$. In the case $\beta \in((r-2 s) / 2,(r-s) / 2)$ one uses in addition the symmetry $h(t)=-h(r-t)$.

3.2. Proof of Theorem 2.3. Our proof of Theorem 2.3] is close in spirit to BL where a remainder term in the Sobolev inequality on bounded domains was found. We first exhibit functions $\psi \in \operatorname{dom} h_{s}$ in the form domain which do not lie in the operator domain but for which the singularity of the distribution (indeed, function) $H_{s} \psi$ at $x=0$ can be calculated explicitly.

Lemma 3.3. Let $0 \leq \chi \leq 1$ be a smooth function on $\mathbb{R}_{+}$of compact support, with $\chi(r)=1$ for $r \leq 1$. Define

$$
\psi_{\lambda}(x):=\chi(|x| / \lambda)|x|^{-\alpha}
$$

for $0<\alpha<(d-2 s) / 2$ and $\lambda>0$. Then $\psi_{\lambda} \in \operatorname{dom} h_{s}$ for $0<s<1$ and, for every $\varepsilon>0$, there exists a $\lambda_{\varepsilon}=\lambda_{\varepsilon}(\alpha, d, s, \chi)$ such that for any $\lambda \geq \lambda_{\varepsilon}$,

$$
\left(\left((-\Delta)^{s}-\mathcal{C}_{s, d}|x|^{-2 s}\right) \psi_{\lambda}\right)(x) \leq-\left(\left|\Phi_{s, d}(\alpha)\right|-\varepsilon\right)|x|^{-\alpha-2 s} \quad \text { for all } x \in \mathcal{B}
$$

in the sense of distributions. Here, $\Phi_{s, d}(\alpha)$ is given in (3.5), and $\mathcal{B}$ denotes the unit ball in $\mathbb{R}^{d}$.

Proof. It is not difficult to show that $\psi_{\lambda} \in H^{s}\left(\mathbb{R}^{d}\right)$, which implies that $\psi_{\lambda} \in \operatorname{dom} h_{s}$. (Consult the proof of Proposition 4.1 for details.) Let $0 \leq \varphi \in C_{0}^{\infty}(\mathcal{B})$. According to (3.4) one has

$$
\left(\psi_{\lambda},\left((-\Delta)^{s}-\mathcal{C}_{s, d}|x|^{-2 s}\right) \varphi\right)=\Phi_{s, d}(\alpha)\left(|x|^{-\alpha-2 s}, \varphi\right)-\left(\tilde{\psi}_{\lambda},(-\Delta)^{s} \varphi\right),
$$

where $\tilde{\psi}_{\lambda}(x):=(1-\chi(|x| / \lambda))|x|^{-\alpha}$. It follows from Lemma 3.1 (with the aid of polarization) that

$$
\left(\tilde{\psi}_{\lambda},(-\Delta)^{s} \varphi\right)=-2 a_{s, d} \iint \frac{\tilde{\psi}_{\lambda}(y) \varphi(x)}{|x-y|^{d+2 s}} d x d y \geq-\rho(\lambda) \int_{\mathbb{R}^{d}} \frac{\varphi(x)}{|x|^{\alpha+2 s}} d x
$$


with

$$
\begin{aligned}
\rho(\lambda) & =\sup _{|x| \leq 1} 2 a_{s, d}|x|^{\alpha+2 s} \int \frac{\tilde{\psi}_{\lambda}(y)}{|x-y|^{d+2 s}} d y \\
& =\sup _{|x| \leq 1 / \lambda} 2 a_{s, d}|x|^{\alpha+2 s} \int \frac{1-\chi(y)}{|y|^{\alpha}|x-y|^{d+2 s}} d y .
\end{aligned}
$$

Note that $\rho(\lambda)$ is finite for $\lambda \geq 1$, and monotone decreasing to 0 as $\lambda \rightarrow \infty$. Hence, for a given $\varepsilon>0$ we can choose $\lambda_{\varepsilon}$ such that $\rho\left(\lambda_{\varepsilon}\right)=\varepsilon$. Since $\Phi_{s, d}(\alpha)$ is negative by Lemma 3.2 we have established (3.9).

Lemma 3.4. Let $1 \leq q<\infty$ and $u \in L^{q}\left(\mathbb{R}^{d}\right)$ be a symmetric decreasing function. Then

$$
\|u\|_{q} \leq q^{-1}|\mathcal{B}|^{-1 / q^{\prime}} \int_{\mathbb{R}^{d}} u(x)|x|^{-d / q^{\prime}} d x
$$

where $|\mathcal{B}|$ is the volume of the unit ball $\mathcal{B}$ in $\mathbb{R}^{d}$, and $1 / q+1 / q^{\prime}=1$.

Proof. First note that (3.11) is true (with equality) if $u$ is the characteristic function of a centered ball. For general $u$ we use the layer cake representation LLo, Thm. 1.13], $u(x)=\int_{0}^{\infty} \chi_{t}(x) d t$, where $\chi_{t}$ is the characteristic function of a centered ball of a certain $t$-dependent radius. Then, by Minkowski's inequality LLO, Thm. 2.4],

$$
\begin{aligned}
\|u\|_{q} & \leq \int_{0}^{\infty}\left\|\chi_{t}\right\|_{q} d t=q^{-1}|\mathcal{B}|^{-1 / q^{\prime}} \int_{0}^{\infty} \int \chi_{t}(x)|x|^{-d / q^{\prime}} d x d t \\
& =q^{-1}|\mathcal{B}|^{-1 / q^{\prime}} \int u(x)|x|^{-d / q^{\prime}} d x,
\end{aligned}
$$

proving (3.11).

Now we give the

Proof of Theorem 2.3. We remark first that we may assume $\Omega$ to be a ball and $u$ to be a spherically symmetric decreasing function. Indeed, passing to the symmetric decreasing rearrangement of $u$ leaves the left side of (2.6) invariant while it decreases the right side. The kinetic energy term on the right side is decreased by virtue of Riesz's rearrangement inequality (compare with [LLO, Thm. 3.7, Lemma 7.17]), and $\int|u|^{2}|x|^{-2 s} d x$ increases [LLO, Thm. 3.4]. Moreover, by scaling we may assume that $\Omega=\mathcal{B}$, the unit ball.

Since $\mathcal{B}$ is bounded, Hölder's inequality implies that it suffices to prove (2.6) for $d /(d-2 s)<q<2 d /(d-2 s)$. For such $q$ let $\alpha:=d / q^{\prime}-2 s$ and note that $0<\alpha<(d-2 s) / 2$. It follows from Lemmas 3.4 and 3.3 that for symmetric decreasing functions $u$ on $\mathcal{B}$

$$
\|u\|_{q} \leq q^{-1}|\mathcal{B}|^{-1 / q^{\prime}} \int_{\mathcal{B}} u(x)|x|^{-d / q^{\prime}} d x \leq 2 q^{-1}|\mathcal{B}|^{-1 / q^{\prime}}\left|\Phi_{s, d}(\alpha)\right|^{-1}\left|\left(u, H_{s} \psi\right)\right| .
$$

Here $\psi=\psi_{\lambda_{\varepsilon}}$ is chosen as in Lemma 3.3, with $\varepsilon=\left|\Phi_{s, d}(\alpha)\right| / 2$. An application of Schwarz's inequality, $\left|\left(u, H_{s} \psi\right)\right|^{2} \leq h_{s}[u] h_{s}[\psi]$, concludes the proof of (2.6).

In Appendix $\mathrm{A}$, we shall give an upper bound on the constant appearing in the Sobolev inequality in the special case of $d=3$ and $s=1 / 2$, which is the case of interest in the application in Subsection 2.2 
3.3. Proof of Corollary 2.5. We will deduce Corollary 2.5 from Theorem 2.3 by a localization argument. For comparison, we first recall the IMS formula in the local case $s=1$. If $\chi_{0}, \ldots, \chi_{n}$ are Lipschitz continuous functions on $\mathbb{R}^{d}$ satisfying $\sum_{j=0}^{n} \chi_{j}^{2} \equiv 1$, then

$$
\begin{aligned}
\int_{\mathbb{R}^{d}} & \left(|\nabla u|^{2}-\frac{(d-2)^{2}}{4} \frac{|u|^{2}}{|x|^{2}}\right) d x \\
& =\sum_{j=0}^{n} \int_{\mathbb{R}^{d}}\left(\left|\nabla\left(\chi_{j} u\right)\right|^{2}-\frac{(d-2)^{2}}{4} \frac{\left|\chi_{j} u\right|^{2}}{|x|^{2}}\right) d x-\int_{\mathbb{R}^{d}} \sum_{j=0}^{n}\left|\nabla \chi_{j}\right|^{2}|u|^{2} d x .
\end{aligned}
$$

The following analogous formula for the non-local case, suggested by Michael Loss, is given in $[\mathrm{LY}]$. The proof is an immediate consequence of Lemma 3.1. For a generalization to the magnetic case, see Lemma B.1 below.

Lemma 3.5. Let $0<s<\min \{1, d / 2\}$ and let $\chi_{0}, \ldots, \chi_{n}$ be Lipschitz continuous functions on $\mathbb{R}^{d}$ satisfying $\sum_{j=0}^{n} \chi_{j}^{2} \equiv 1$. Then

$$
h_{s}[u]=\sum_{j=0}^{n} h_{s}\left[\chi_{j} u\right]-(u, L u), \quad u \in C_{0}^{\infty}\left(\mathbb{R}^{d}\right),
$$

where $L$ is the bounded operator with integral kernel

$$
L(x, y):=a_{s, d}|x-y|^{-d-2 s} \sum_{j=0}^{n}\left(\chi_{j}(x)-\chi_{j}(y)\right)^{2} .
$$

Let us recall the following (non-critical) Sobolev embedding theorem, which is easy to prove. (Cf., e.g., the proof of [LLO, Thm. 8.5].) If $s<d / 2$ and $2 \leq q<$ $2^{*}=2 d /(d-2 s)$, then $H^{s}\left(\mathbb{R}^{d}\right) \subset L^{q}\left(\mathbb{R}^{d}\right)$ and

$$
\|u\|_{q}^{2} \leq S_{q, d, s}\left(\left\|(-\Delta)^{s / 2} u\right\|^{2}+\|u\|^{2}\right), \quad u \in H^{s}\left(\mathbb{R}^{d}\right) .
$$

In combination with the localization Lemma 3.5 this allows us to give the

Proof of Corollary 2.5. Let $\chi_{0}, \chi_{1}$ be smooth functions on $\mathbb{R}^{d}$ with $\chi_{0}^{2}+\chi_{1}^{2} \equiv 1$ such that $\chi_{0}(x)=0$ if $|x| \geq 1$ and $\chi_{1}(x)=0$ if $|x| \leq 1 / 2$. Let $2 \leq q<2 d /(d-2 s)$. Then, by Theorem 2.3,

$$
\left\|\chi_{0} u\right\|_{q}^{2} \leq C_{q, d, s}|\mathcal{B}|^{2\left(\frac{1}{q}-\frac{1}{2^{*}}\right)} h_{s}\left[\chi_{0} u\right],
$$

and by (3.14)

$$
\begin{aligned}
\left\|\chi_{1} u\right\|_{q}^{2} & \leq S_{q, d, s}\left(\left\|(-\Delta)^{s / 2}\left(\chi_{1} u\right)\right\|^{2}+\left\|\chi_{1} u\right\|^{2}\right) \\
& \leq S_{q, d, s}\left(h_{s}\left[\chi_{1} u\right]+\left(2^{2 s} \mathcal{C}_{s, d}+1\right)\left\|\chi_{1} u\right\|^{2}\right) .
\end{aligned}
$$

Hence Corollary 2.5 follows from Lemma 3.5 noting that $L$ is a bounded operator.

\section{Ground state Representation}

Equation (3.4) and Lemma 3.2 suggest that the function $|x|^{-(d-2 s) / 2}$ is a 'generalized ground state' of the operator $H_{s}$. Our next goal is to establish a ground 
state representation. Let us recall the analogous formula in the 'local' case $s=1$. If $d \geq 3$ and $v(x)=|x|^{(d-2) / 2} u(x)$, then

$$
\int_{\mathbb{R}^{d}}\left(|\nabla u|^{2}-\frac{(d-2)^{2}}{4} \frac{|u|^{2}}{|x|^{2}}\right) d x=\int_{\mathbb{R}^{d}}|\nabla v|^{2} \frac{d x}{|x|^{d-2}} .
$$

The corresponding formula in the non-local case $0<s<1$ is more complicated but close in spirit. It was derived some years ago by Michael Loss (unpublished notes) for the relativistic case $s=1 / 2$ and $d=3$. This representation can be independently useful Be2].

Proposition 4.1 (Ground State Representation). Let $0<s<\min \{1, d / 2\}$. If $u \in C_{0}^{\infty}\left(\mathbb{R}^{d} \backslash\{0\}\right)$ and $v(x)=|x|^{(d-2 s) / 2} u(x)$, then

$$
h_{s}[u]=a_{s, d} \int_{\mathbb{R}^{d}} \int_{\mathbb{R}^{d}} \frac{|v(x)-v(y)|^{2}}{|x-y|^{d+2 s}} \frac{d x}{|x|^{(d-2 s) / 2}} \frac{d y}{|y|^{(d-2 s) / 2}},
$$

with $a_{s, d}$ given in (3.2).

Proof. Let $0<\alpha<(d-2 s) / 2$. We shall prove that if $u \in C_{0}^{\infty}\left(\mathbb{R}^{d} \backslash\{0\}\right)$ and $v_{\alpha}(x):=|x|^{\alpha} u(x)$, then

$$
\begin{gathered}
\int_{\mathbb{R}^{d}}|\xi|^{2 s}|\hat{u}(\xi)|^{2} d \xi-\left(\mathcal{C}_{s, d}+\Phi_{s, d}(\alpha)\right) \int_{\mathbb{R}^{d}}|x|^{-2 s}|u(x)|^{2} d x \\
=a_{s, d} \int_{\mathbb{R}^{d}} \int_{\mathbb{R}^{d}} \frac{\left|v_{\alpha}(x)-v_{\alpha}(y)\right|^{2}}{|x-y|^{d+2 s}} \frac{d x}{|x|^{\alpha}} \frac{d y}{|y|^{\alpha}} .
\end{gathered}
$$

The proposition follows by letting $\alpha \rightarrow(d-2 s) / 2$. Indeed, the constant in front of the second integral on the left side then converges to $\mathcal{C}_{s, d}$, according to Lemma 3.2 , By splitting the integral into four regions according to the support of $u$, it is easy to see that the right side is continuous in $\alpha$ and converges to the right side of (4.2).

For the proof of (4.3) we can suppose that the support of $u$ is in the unit ball. We shall first prove the equality for mollified versions of $|x|^{-\alpha}$, namely functions $\omega_{n}(x)=|x|^{-\alpha} \chi(x / n)$, where $\chi \in C_{0}^{\infty}\left(\mathbb{R}^{d}\right)$ with $\chi(x)=1$ for $|x| \leq 1$.

Let us first show that $\omega_{n} \in H^{s}\left(\mathbb{R}^{d}\right)$. It is clearly in $L^{2}\left(\mathbb{R}^{d}\right)$, hence it suffices to establish that $(-\Delta)^{s / 2} \omega_{n} \in L^{2}\left(\mathbb{R}^{d}\right)$. According to [LLO, Thm. 5.9] the Fourier transform of $\omega_{n}$ is given by the convolution of $\hat{\chi}$ and $|\xi|^{\alpha-d}$. Since $\chi$ is assumed to be smooth, $\widehat{\chi}$ decays faster than any power of $|\xi|$. It is then easy to see that $\widehat{\omega}_{n}$ decays like $|\xi|^{\alpha-d}$, and hence $|\xi|^{s} \widehat{\psi} \in L^{2}\left(\mathbb{R}^{d}\right)$.

By polarization in Lemma 3.1, we get for any $f$ and $g$ in $H^{s}\left(\mathbb{R}^{d}\right)$,

$$
\int_{\mathbb{R}^{d}}|\xi|^{2 s} \overline{\widehat{f}(\xi)} \widehat{g}(\xi) d \xi=a_{s, d} \iint_{\mathbb{R}^{d} \times \mathbb{R}^{d}}(\overline{f(x)}-\overline{f(y)})(g(x)-g(y)) \frac{d x d y}{|x-y|^{d+2 s}} .
$$

We apply this formula to $g(x)=\omega_{n}(x)$ and $f(x)=|u(x)|^{2} / \omega_{n}(x)=|u(x)|^{2}|x|^{\alpha}$. In this case, the right side of (4.4) is given by

$$
a_{s, d} \iint_{\mathbb{R}^{d} \times \mathbb{R}^{d}}\left(|u(x)-u(y)|^{2}-\left|\frac{u(x)}{\omega_{n}(x)}-\frac{u(y)}{\omega_{n}(y)}\right|^{2} \omega_{n}(x) \omega_{n}(y)\right) \frac{d x d y}{|x-y|^{d+2 s}} .
$$

Note that $u(x) / \omega_{n}(x)=u(x)|x|^{\alpha}=v_{\alpha}(x)$ is independent of $n$, and is a $C_{0}^{\infty}$ function since the origin is not in the support of $u$ by assumption. By dominated 
convergence, (4.5) converges to

$$
a_{s, d} \iint_{\mathbb{R}^{d} \times \mathbb{R}^{d}}\left(|u(x)-u(y)|^{2}-\left|v_{\alpha}(x)-v_{\alpha}(y)\right|^{2}|x|^{-\alpha}|y|^{-\alpha}\right) \frac{d x d y}{|x-y|^{d+2 s}}
$$

as $n \rightarrow \infty$. The left side of (4.4) can be written as (compare with (3.3))

$$
(2 \pi)^{d / 2} \frac{b_{d-\alpha}}{b_{\alpha}} \iint_{\mathbb{R}^{d} \times \mathbb{R}^{d}}|\xi|^{2 s} \overline{\widehat{f}(\xi)} n^{d} \widehat{\chi}\left(n\left(\xi-\xi^{\prime}\right)\right)\left|\xi^{\prime}\right|^{\alpha-d} d \xi d \xi^{\prime} .
$$

Since $\widehat{f}$ decays faster than polynomially, $|\cdot|{ }^{2 s} \widehat{f} \in L^{p}\left(\mathbb{R}^{d}\right)$ for any $1 \leq p<\infty$. Hence its convolution with the approximate $\delta$-function $(2 \pi)^{d / 2} n^{d} \widehat{\chi}(n \cdot)$ converges to $|\cdot|^{2 s} \widehat{f}$ strongly in any $L^{p}$, for $1 \leq p<\infty$ [LLo, Thm. 2.16]. Therefore, (4.7) converges to

$$
\frac{b_{d-\alpha}}{b_{\alpha}} \int_{\mathbb{R}^{d}}|\xi|^{2 s+\alpha-d} \overline{\widehat{f}(\xi)} d \xi=\frac{b_{\alpha+2 s} b_{d-\alpha}}{b_{d-\alpha-2 s} b_{\alpha}} \int_{\mathbb{R}^{d}}|u(x)|^{2}|x|^{-2 s} d x .
$$

Here we used (3.3) again. The equality of (4.6) and (4.8) proves (4.3).

Remark 4.2. From the representation (4.2) we immediately recover Hardy's inequalities (2.1) in the case $s<\min \{1, d / 2\}$. Moreover, we see that equality cannot be attained (for a non-zero function), as we remarked in the Introduction. From (4.2), it is also easy to see that the constant $\mathcal{C}_{s, d}$ is sharp. For this, consider a sequence of functions $u_{n}$, supported in $\mathcal{B}$, approximating $|x|^{-(d-2 s) / 2}$ close to the origin in a suitable sense. The right side of (4.2) remains finite in the limit $n \rightarrow \infty$, whereas $\int\left|u_{n}(x)\right|^{2}|x|^{-2 s} d x$ diverges.

\section{Proof of the Hardy-Lieb-Thirring inequalities}

This section contains the proof of our main result in Theorem 2.1. Here we consider only the non-magnetic case $A=0$; the extension to non-zero $A$ will be straightforward given the necessary diamagnetic inequalities which we derive in Section 6. We explain the necessary modifications in the proof of Theorem 2.1] in Subsection 6.3.

The ground state representation (4.2) suggests that it is more natural to regard $h_{s}[u]$ as a function of $v$ given by $v(x)=|x|^{(d-2 s) / 2} u(x)$. In terms of this function $v$, the Sobolev-Hardy inequality in (2.7) can be formulated in the weighted space with measure $|x|^{-\beta} d x$, where $\beta=q(d-2 s) / 2$. Namely,

$$
\|v\|_{L^{q}\left(\mathbb{R}^{d},|x|^{-\beta} d x\right)}^{2} \leq C_{q, d, s}^{\prime}\left(v, B_{\beta} v\right)_{L^{2}\left(\mathbb{R}^{d},|x|^{-\beta} d x\right)},
$$

where $B_{\beta}$ is the operator on $L^{2}\left(\mathbb{R}^{d},|x|^{-\beta} d x\right)$ defined by the quadratic form

$$
\left(v, B_{\beta} v\right)_{L^{2}\left(\mathbb{R}^{d},|x|^{-\beta} d x\right)}=h_{s}\left[|x|^{-(d-2 s) / 2} v\right]+\left\||x|^{-(d-2 s) 2} v\right\|_{L^{2}\left(\mathbb{R}^{d}, d x\right)}^{2} .
$$

We suppress the dependence on $s$ in $B_{\beta}$ for simplicity. Note that the right side of (5.2) is independent of $\beta$. The dependence of $B_{\beta}$ on $\beta$ comes from the measure $|x|^{-\beta} d x$ of the underlying $L^{2}$ space, which is determined by the value of $q$ in the Sobolev inequality (5.1) as $\beta=q(d-2 s) / 2$. We emphasize again that the choice of the weight $|x|^{-(d-2 s) / 2}$ on the right side of (5.2) is determined by the ground state representation (4.2).

The proof of Theorem 2.1 proceeds in the following steps.

- From the ground state representation (4.2), we will deduce that $B_{\beta}$ satisfies the Beurling-Deny criteria, which implies that $e^{-t B_{\beta}}$ is a contraction on $L^{1}\left(\mathbb{R}^{d},|x|^{-\beta} d x\right)$ (Subsection 5.1). 
- Together with the Sobolev-Hardy inequality (5.1) this yields a bound on the kernel of $e^{-t B_{\beta}}$ via Nash's method (Subsection 5.2).

- This bound on the heat kernel can then be translated into a LT bound in the spirit of [L1] (Subsection 5.3).

5.1. Contraction property of $B_{\beta}$. Let $\mathfrak{H}_{\beta}:=L^{2}\left(\mathbb{R}^{d},|x|^{-\beta} d x\right)$. We assume that $d-2 s<\beta<d$, which corresponds to $2<q<2^{*}$ in (5.1). The quadratic form

$$
b_{\beta}[v]:=h_{s}\left[|x|^{-(d-2 s) / 2} v\right]+\left\||x|^{-(d-2 s) / 2} v\right\|^{2},
$$

considered in the Hilbert space $\mathfrak{H}_{\beta}$, is non-negative and closable on $C_{0}^{\infty}\left(\mathbb{R}^{d} \backslash\{0\}\right)$, and hence generates a self-adjoint operator $B_{\beta}$ in $\mathfrak{H}_{\beta}$.

We shall deduce some positivity properties of the operator $\exp \left(-t B_{\beta}\right)$. By Proposition 4.1 the quadratic form $b_{\beta}$ satisfies

(1) if $v, w \in \operatorname{dom} b_{\beta}$ are real-valued, then $b_{\beta}[v+i w]=b_{\beta}[v]+b_{\beta}[w]$,

(2) if $v \in \operatorname{dom} b_{\beta}$ is real-valued, then $|v| \in \operatorname{dom} b_{\beta}$ and $b_{\beta}[|v|] \leq b_{\beta}[v]$,

(3) if $v \in \operatorname{dom} b_{\beta}$ is non-negative, then $\min (v, 1) \in \operatorname{dom} b_{\beta}$ and $b_{\beta}[\min (v, 1)] \leq$ $b_{\beta}[v]$.

By a theorem of Beurling-Deny (see [D, Section 1.3] or [ReSi2, Section XIII.12]) this implies that $\exp \left(-t B_{\beta}\right)$ is positivity-preserving and a contraction in $L^{1}\left(\mathbb{R}^{d},|x|^{-\beta} d x\right)$. That is, it maps non-negative functions into non-negative functions, and it decreases $L^{1}$-norms.

5.2. Heat kernel estimate. From the contraction property derived above, we will deduce a pointwise bound on the heat kernel, i.e., on the kernel of the integral operator $\exp \left(-t B_{\beta}\right)$. We emphasize that this kernel is defined by

$$
\left(\exp \left(-t B_{\beta}\right) v\right)(x)=\int_{\mathbb{R}^{d}} \exp \left(-t B_{\beta}\right)(x, y) v(y) \frac{d y}{|y|^{\beta}} .
$$

We shall use the Sobolev inequality (5.1) for this purpose.

Proposition 5.1. Let $d-2 s<\beta<d$. Then $\exp \left(-t B_{\beta}\right)$ is an integral operator on $\mathfrak{H}_{\beta}$ and its kernel satisfies

$$
0 \leq \exp \left(-t B_{\beta}\right)(x, y) \leq K_{\beta, d, s} t^{-p} \quad t>0, x, y \in \mathbb{R}^{d},
$$

where $p:=\beta /(\beta-d+2 s)$. The constant can be chosen to be $K_{\beta, d, s}:=\left(p C_{q, d, s}^{\prime}\right)^{p}$ where $C_{q, d, s}^{\prime}$ is the constant from Corollary 2.5 with $q:=2 \beta /(d-2 s)$.

Proof. Let $\theta:=(q-2) /(q-1) \in(0,1)$. Then Hölder's inequality and Corollary 2.5 yield for any $v \in C_{0}^{\infty}\left(\mathbb{R}^{d} \backslash\{0\}\right)$

$$
\begin{aligned}
\|v\|_{L^{2}\left(\mathbb{R}^{d},|x|^{-\beta} d x\right)}^{2} & \leq\|v\|_{L^{q}\left(\mathbb{R}^{d},|x|^{-\beta} d x\right)}^{1-\theta}\|v\|_{L^{1}\left(\mathbb{R}^{d},|x|^{-\beta} d x\right)}^{\theta} \\
& \leq C_{q, d, s}^{\prime(1-\theta) / 2} b_{\beta}[v]^{(1-\theta) / 2}\|v\|_{L^{1}\left(\mathbb{R}^{d},|x|^{-\beta} d x\right)}^{\theta} .
\end{aligned}
$$

Equivalently, if $p$ is as in the proposition, then

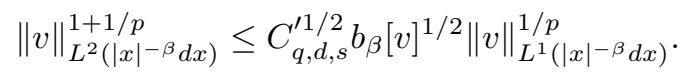

This is a Nash-type inequality in $\mathbb{R}^{d}$ with measure $|x|^{-\beta} d x$. By Nash's argument (see [LLo, Theorem 8.16] or [D, Section 2.4]) this implies that $\exp \left(-t B_{\beta}\right)$ is an integral operator with kernel satisfying (15.3), with the constant $K_{\beta, d, s}$ given in the proposition. For the sake of completeness we sketch the proof of this claim in Appendix D below. 
Remark 5.2. In the above argument we used the Nash-type inequality (5.4) which we had deduced from the Sobolev-type inequality (2.7). The heat kernel bound (5.3) would actually follow directly from the latter inequality by [D, Thm. 2.4.2]. However, we preferred the simplicity of the above argument, yielding in addition an explicit constant.

5.3. Proof of Theorem 2.1. Step 1. As a first step, we seek an upper bound on the number of eigenvalues below $-\tau$ of the operator $H_{s}-V$, which we denote by $N\left(-\tau, H_{s}-V\right)$. By the variational principle we may assume that $V \geq 0$. Then the Birman-Schwinger principle (see, e.g., ReSi2 ) implies that for any increasing non-negative function $F$ on $(0, \infty)$

$$
N\left(-1, H_{s}-V\right) \leq F(1)^{-1} \operatorname{tr} F\left(V^{1 / 2}\left(H_{s}+I\right)^{-1} V^{1 / 2}\right) .
$$

Let $\mathcal{U}: L_{2}\left(\mathbb{R}^{d}\right) \rightarrow \mathfrak{H}_{\beta}$ be the unitary operator which maps $u \mapsto|x|^{\beta / 2} u$. Then

$$
V^{1 / 2}\left(H_{s}+I\right)^{-1} V^{1 / 2}=\mathcal{U}^{*} W_{\beta}^{1 / 2} B_{\beta}^{-1} W_{\beta}^{1 / 2} \mathcal{U}
$$

where $W_{\beta}$ is the multiplication operator on $\mathfrak{H}_{\beta}$ which multiplies by the function $W_{\beta}(x):=|x|^{\beta+2 s-d} V(x)$. Therefore

$$
\operatorname{tr} F\left(V^{1 / 2}\left(H_{s}+I\right)^{-1} V^{1 / 2}\right)=\operatorname{tr}_{\mathfrak{H}_{\beta}} F\left(W_{\beta}^{1 / 2} B_{\beta}^{-1} W_{\beta}^{1 / 2}\right) .
$$

We need the following trace estimate.

Proposition 5.3. Let $f$ be a non-negative convex function on $[0, \infty)$, growing polynomially at infinity and vanishing near the origin, and let

$$
F(\lambda):=\int_{0}^{\infty} f(\mu) e^{-\mu / \lambda} \mu^{-1} d \mu, \quad \lambda>0 .
$$

Then for any $d-2 s<\beta<d$ and any multiplication operator $W \geq 0$

$$
\operatorname{tr}_{\mathfrak{H}_{\beta}} F\left(W^{1 / 2} B_{\beta}^{-1} W^{1 / 2}\right) \leq \int_{0}^{\infty} \int_{\mathbb{R}^{d}} \exp \left(-t B_{\beta}\right)(x, x) f(t W(x)) \frac{d x}{|x|^{\beta}} \frac{d t}{t} .
$$

Note that the heat kernel $\exp \left(-t B_{\beta}\right)(x, y)$ is well defined on the diagonal $x=y$ by the semigroup property. Namely,

$$
\exp \left(-t B_{\beta}\right)(x, x)=\int\left|\exp \left(-t B_{\beta} / 2\right)(x, y)\right|^{2}|y|^{-\beta} d y \text {. }
$$

For the proof of Proposition 5.3 one follows the proof of the CLR bound in L1] (see also $\mathrm{Si2}$ and $\underline{\mathrm{RoSo}}$ ). As in the latter paper Trotter's product formula can be used in place of path integrals. For details we refer to Appendix E

We shall now assume that $F$ has the special form (5.7) in order to apply the trace estimate from Proposition 5.3. Given $d-2 s<\beta<d$ and $p=\beta /(\beta-d+2 s)$, Proposition 5.1 implies that

$$
\begin{aligned}
\int_{0}^{\infty} & \int_{\mathbb{R}_{d}} \exp \left(-t B_{\beta}\right)(x, x) f\left(t W_{\beta}(x)\right) \frac{d x}{|x|^{\beta}} \frac{d t}{t} \\
& \leq K_{\beta, d, s} \int_{0}^{\infty} \int_{\mathbb{R}^{d}} t^{-p} f\left(t W_{\beta}(x)\right) \frac{d x}{|x|^{\beta}} \frac{d t}{t} \\
& =K_{\beta, d, s}\left(\int_{\mathbb{R}^{d}} W_{\beta}(x)^{p} \frac{d x}{|x|^{\beta}}\right)\left(\int_{0}^{\infty} t^{-p-1} f(t) d t\right) .
\end{aligned}
$$


Note that $W_{\beta}(x)^{p}=V(x)^{p}|x|^{\beta}$. We conclude that for any $d / 2 s<p<\infty$,

$$
N\left(-1, H_{s}-V\right) \leq K_{p, d, s}^{\prime} \int_{\mathbb{R}^{d}} V(x)_{+}^{p} d x
$$

where the constant is given by

$$
K_{p, d, s}^{\prime}=K_{\beta, d, s} \inf _{f} F(1)^{-1}\left(\int_{0}^{\infty} t^{-p-1} f(t) d t\right) .
$$

Here $\beta=p(d-2 s) /(p-1)$, and the infimum runs over all admissible functions $f$ from Proposition 5.3. In order to obtain an explicit upper bound one may choose $f(x):=(x-a)+$ and minimize over $a>0$.

Step 2. Now we use the idea of [LTh to deduce (2.5) from (5.9). Fix $\gamma>0$ and choose some $d /(2 s)<p<\gamma+d /(2 s)$. First we note that by scaling we have, for any $\tau>0$,

$$
N\left(-\tau, H_{s}-V\right)=N\left(-1, H_{s}-V_{\tau}\right)
$$

where $V_{\tau}(x):=\tau^{-1} V\left(\tau^{-1 / 2 s} x\right)$. In view of (5.9) this yields

$$
N\left(-\tau, H_{s}-V\right) \leq K_{p, d, s}^{\prime} \tau^{-p+d / 2 s} \int_{\mathbb{R}^{d}} V(x)_{+}^{p} d x
$$

Now, for any fixed $0<\sigma<1$, one has by the variational principle

$$
N\left(-\tau, H_{s}-V\right) \leq N\left(-(1-\sigma) \tau, H_{s}-(V-\sigma \tau)_{+}\right) .
$$

Hence, by (5.10),

$$
\begin{aligned}
\operatorname{tr}\left(H_{s}-V\right)_{-}^{\gamma} & =\gamma \int_{0}^{\infty} N\left(-\tau, H_{s}-V\right) \tau^{\gamma-1} d \tau \\
& \leq \gamma K_{p, d, s}^{\prime}(1-\sigma)^{-p+d / 2 s} \int_{0}^{\infty} \int_{\mathbb{R}^{d}}(V(x)-\sigma \tau)_{+}^{p} d x \tau^{\gamma-p+d / 2 s-1} d \tau
\end{aligned}
$$

We change the order of integration and calculate the $\tau$-integral first. For fixed $x \in \mathbb{R}^{d}$,

$$
\int_{0}^{\infty}(V(x)-\sigma \tau)_{+}^{p} \tau^{\gamma-p+d / 2 s-1} d \tau=\sigma^{-\gamma-d / 2 s+p} V(x)_{+}^{\gamma+d / 2 s} B(\gamma+d / 2 s-p, p+1) .
$$

Here, $B$ denotes the Beta-function $B(a, b)=\Gamma(a) \Gamma(b) / \Gamma(a+b)$. Minimization over $\sigma \in(0,1)$ and $p \in(d / 2 s, \gamma+d / 2 s)$ yields

$$
\operatorname{tr}\left(H_{s}-V\right)_{-}^{\gamma} \leq C_{d, s}(\gamma) \int_{\mathbb{R}^{d}} V(x)_{+}^{\gamma+d / 2 s} d x
$$

with

$$
\begin{aligned}
C_{d, s}(\gamma):=\min _{d / 2 s<p<\gamma+d / 2 s}\{ & \gamma^{\gamma+1} K_{p, d, s}^{\prime} B(\gamma+d / 2 s-p, p+1) \\
& \left.\times(\gamma+d / 2 s-p)^{-\gamma-d / 2 s+p}(p-d / 2 s)^{-p+d / 2 s}\right\} .
\end{aligned}
$$

This concludes the proof of Theorem 2.1 in the case $A=0$. 


\section{Extension to MAGNETIC FIELDS}

In this section we prove certain diamagnetic inequalities which allow us to extend the proof of Theorem 2.1 in the previous section to the case of non-zero magnetic fields. The main idea is contained in Proposition 6.1 in Subsection 6.1. Subsection 6.2 contains some technical refinements we will need. In Subsection 6.3 we describe the necessary modifications in the proof of Theorem 2.1 to include magnetic fields. Subsection 6.4 is devoted to the special case $s=1$.

Throughout this section we shall assume that $A \in L_{\mathrm{loc}}^{2}\left(\mathbb{R}^{d} ; \mathbb{R}^{d}\right)$ and that $d \geq 2$. Note that in $d=1$ any magnetic vector potential can be removed by a gauge transformation.

In $\mathfrak{H}_{\beta}=L^{2}\left(\mathbb{R}^{d},|x|^{-\beta} d x\right)$ consider the quadratic form

$$
b_{\beta, A}[v]:=h_{s, A}\left[|x|^{-(d-2 s) / 2} v\right]+\left\||x|^{-(d-2 s) / 2} v\right\|^{2}, \quad v \in C_{0}^{\infty}\left(\mathbb{R}^{d} \backslash\{0\}\right) .
$$

In Appendix $\mathrm{C}$, we show that $b_{\beta, A}$ is closable and hence defines a self-adjoint operator $B_{\beta, A}$ in $\mathfrak{H}_{\beta}$. Our goal in this section is to show that $\exp \left(-t B_{\beta, A}\right)$ is an integral operator, whose kernel satisfies

$$
\left|\exp \left(-t B_{\beta, A}\right)(x, y)\right| \leq \exp \left(-t B_{\beta}\right)(x, y) .
$$

6.1. An initial inequality. We consider weighted magnetic operators $\omega|D-A|^{2 s} \omega$ where $A \in L_{\text {loc }}^{2}\left(\mathbb{R}^{d}\right)$ and $\omega>0$ with $\omega+\omega^{-1} \in L^{\infty}\left(\mathbb{R}^{d}\right)$. This is a self-adjoint operator in $L^{2}\left(\mathbb{R}^{d}\right)$ with form domain $\omega^{-1} \operatorname{dom}|D-A|^{s}$. It satisfies the following diamagnetic inequality.

Proposition 6.1 (Weighted diamagnetic inequality). Let $d \geq 2$ and $0<s \leq 1$. Assume that $A \in L_{\mathrm{loc}}^{2}\left(\mathbb{R}^{d}\right)$ and that $\omega>0$ with $\omega+\omega^{-1} \in L^{\infty}\left(\mathbb{R}^{d}\right)$. Then for all $u \in L^{2}\left(\mathbb{R}^{d}\right)$ and all $t \geq 0$ one has

$$
\left|\exp \left(-t \omega|D-A|^{2 s} \omega\right) u\right| \leq \exp \left(-t \omega(-\Delta)^{s} \omega\right)|u|
$$

Proof. First note that the assertion is true in the case $\omega \equiv 1$, i.e.,

$$
\left|\exp \left(-t|D-A|^{2 s}\right) u\right| \leq \exp \left(-t(-\Delta)^{s}\right)|u|
$$

Indeed, for $s=1$ this inequality is proved in Si1] for all $A \in L_{\mathrm{loc}}^{2}\left(\mathbb{R}^{d}\right)$. The general case $0<s<1$ follows from the fact that the function $\lambda \mapsto e^{-\lambda^{s}}$ is completely monotone (i.e., its derivatives are alternating in sign) and hence is the Laplace transform of a positive measure by Bernstein's theorem [Do. This reduces the problem to the case $s=1$.

Now assume that $\omega$ is as in the proposition and write $M_{A}:=\omega|D-A|^{2 s} \omega$. In view of the general relation

$$
\exp \left(-t M_{A}\right)=\operatorname{s}_{n \rightarrow \infty}\left(I+n^{-1} t M_{A}\right)^{-n}
$$

it suffices to prove the inequality $\left|\left(M_{A}+\tau\right)^{-1} u\right| \leq\left(M_{0}+\tau\right)^{-1}|u|$. But since $\left(M_{A}+\tau\right)^{-1}=\omega^{-1}\left(|D-A|^{2 s}+V\right)^{-1} \omega^{-1}$ with $V:=\tau \omega^{-2}$ it suffices to prove $\left|\left(|D-A|^{2 s}+V\right)^{-1} u\right| \leq\left(-\Delta^{s}+V\right)^{-1}|u|$. In view of the relation 'inverse' to (6.5),

$$
\left(|D-A|^{2 s}+V\right)^{-1}=\int_{0}^{\infty} \exp \left(-t\left(|D-A|^{2 s}+V\right)\right) d t
$$

the assertion follows from (6.4) and Trotter's product formula. 
Remark 6.2. We note that the diamagnetic inequality in the form (6.4) implies (2.3). This follows by integrating the square of (6.4) and evaluating the derivative with respect to $t$ at $t=0$.

6.2. A refined inequality. In order to prove the desired inequality (6.2), we have to extend Proposition 6.1 in two directions. First, we want to use the singular weight $\omega(x)=|x|^{\alpha}, 0<\alpha<s$, and second, we want to replace the operator $|D-A|^{2 s}$ by $H_{s, A}+I$, i.e., we want to subtract the Hardy term.

Recall that $B_{\beta, A}$ was defined by (6.1). The main result of this section is the following.

Proposition 6.3 (Weighted diamagnetic inequality, second version). Let $d \geq 2$ and $d-2 s<\beta<d$. Assume that $A \in L_{\mathrm{loc}}^{2}\left(\mathbb{R}^{d}\right)$. Then for all $v \in \mathfrak{H}_{\beta}$ one has

$$
\left|\exp \left(-t B_{\beta, A}\right) v\right| \leq \exp \left(-t B_{\beta}\right)|v| .
$$

It follows from Proposition 5.1 that $\exp \left(-t B_{\beta}\right)$ is an integral operator that maps $L^{1}\left(\mathbb{R}^{d},|x|^{-\beta} d x\right)$ to $L^{\infty}\left(\mathbb{R}^{d},|x|^{-\beta} d x\right)$. Hence (6.6) implies that the same is true for $\exp \left(-t B_{\beta, A}\right)$. Moreover, the kernels are related by the inequality (6.2).

In the course of the proof we will need the following approximation result.

Lemma 6.4. Let $T_{n}, T$ be closed, densely defined operators in a Hilbert space $\mathfrak{H}$ with $T_{n} T_{n}^{*} \rightarrow T T^{*}$ in strong resolvent sense. Assume that there is a set $\mathcal{D} \subset \bigcap \operatorname{dom} T_{n} \cap$ $\operatorname{dom} T$, dense in $\mathfrak{H}$, such that $T_{n} \varphi \rightarrow T \varphi$ for all $\varphi \in \mathcal{D}$. Then $T_{n}^{*} T_{n} \rightarrow T^{*} T$ in strong resolvent sense.

Proof. For $\gamma>0$ and $\varphi, \psi \in \mathcal{D}$ one has

$$
\gamma\left(\varphi,\left(T_{n}^{*} T_{n}+\gamma\right)^{-1} \psi\right)=(\varphi, \psi)-\left(T_{n} \varphi,\left(T_{n} T_{n}^{*}+\gamma\right)^{-1} T_{n} \psi\right) .
$$

By assumption the right side converges to

$$
(\varphi, \psi)-\left(T \varphi,\left(T T^{*}+\gamma\right)^{-1} T \psi\right)=\gamma\left(\varphi,\left(T^{*} T+\gamma\right)^{-1} \psi\right),
$$

which proves that $T_{n}^{*} T_{n} \rightarrow T^{*} T$ in weak resolvent sense. However, the latter is the same as in strong resolvent sense; see [ReSi1, Problem VIII.20].

Proof. Step 1. Again consider the unitary transformation $\mathcal{U}: L^{2}\left(\mathbb{R}^{d}\right) \rightarrow \mathfrak{H}_{\beta}$, which maps $u \mapsto|x|^{\beta / 2} u$. Then

$$
Q_{\beta, A}:=\mathcal{U}^{*} B_{\beta, A} \mathcal{U}
$$

is a self-adjoint operator in the unweighted space $L^{2}\left(\mathbb{R}^{d}\right)$, whose quadratic form is given by

$$
q_{\beta, A}[u]:=h_{s, A}\left[|x|^{\alpha} u\right]+\left\||x|^{\alpha} u\right\|^{2}, \quad \alpha=(\beta+2 s-d) / 2 .
$$

Equation (6.6) is equivalent to

$$
\left|\exp \left(-t Q_{\beta, A}\right) u\right| \leq \exp \left(-t Q_{\beta, 0}\right)|u|
$$

for $u \in L^{2}\left(\mathbb{R}^{d}\right)$.

Step 2. We begin by considering the case where the 'potential terms' in the definition of $Q_{\beta, A}$ are absent. More precisely, we consider the operator $M_{\beta, A}$ in $L^{2}\left(\mathbb{R}^{d}\right)$ generated by the quadratic form $\left\||D-A|^{s}|x|^{\alpha} u\right\|^{2}$ on $C_{0}^{\infty}\left(\mathbb{R}^{d} \backslash\{0\}\right)$. We shall prove that for all $u \in L^{2}\left(\mathbb{R}^{d}\right)$ one has

$$
\left|\exp \left(-t M_{\beta, A}\right) u\right| \leq \exp \left(-t M_{\beta, 0}\right)|u| .
$$


Let $\omega_{n}$ be a family of smooth positive functions which decrease monotonically to $|x|^{\alpha}$ and agree with this function outside a ball a radius $n^{-1}$. Similarly, for fixed $n$ let $\omega_{n, m}$ be a family of smooth positive and bounded functions which increase monotonically to $\omega_{n}$ and agree with this function inside a ball a radius $m$.

The operators $|D-A|^{s} \omega_{n}$ and $|D-A|^{s} \omega_{n, m}$ are easily seen to be closable on $C_{0}^{\infty}\left(\mathbb{R}^{d} \backslash\{0\}\right.$ ) (as in Appendix C) and we denote their closures by $T_{n}$ and $T_{n, m}$, respectively. One finds that $C_{0}^{\infty}\left(\mathbb{R}^{d}\right) \subset \operatorname{dom} T_{n}^{*}$ with $T_{n}^{*} v=\omega_{n}|D-A|^{s} v$ for $v \in$ $C_{0}^{\infty}\left(\mathbb{R}^{d}\right)$, and similarly for $T_{n, m}^{*}$. By construction of $\omega_{n, m}$ the operators $T_{n, m} T_{n, m}^{*}$ are monotonically increasing as $m \rightarrow \infty$ and hence converge in strong resolvent sense to $T_{n} T_{n}^{*}$ by [ReSi1, Thm. S.14]. Noting that $T_{n, m} \varphi \rightarrow T_{n} \varphi$ for any $\varphi \in$ $C_{0}^{\infty}\left(\mathbb{R}^{d} \backslash\{0\}\right)$ we conclude from Lemma 6.4 that $T_{n, m}^{*} T_{n, m} \rightarrow T_{n}^{*} T_{n}$ in strong resolvent sense. One checks that $T_{n, m}^{*} T_{n, m}$ coincides with the operator $\omega_{n, m} \mid D-$ $\left.A\right|^{2 s} \omega_{n, m}$ from Subsection 6.1 and satisfies a diamagnetic inequality by Proposition 6.1. By the strong resolvent convergence the diamagnetic inequality is also valid for $T_{n}^{*} T_{n}$. Now we repeat the argument for $n \rightarrow \infty$ where we have monotone convergence from above. We apply $\left[\mathrm{D}\right.$, Thm. 1.2.3] noting that $C_{0}^{\infty}\left(\mathbb{R}^{d} \backslash\{0\}\right)$ is a form core for all the operators involved, and conclude that $T_{n}^{*} T_{n} \rightarrow M_{\alpha, A}$ in strong resolvent sense. This proves the diamagnetic inequality (6.10).

Step 3. Now we use another approximation argument to include the Hardy term. We define $R_{\beta, A}$ via the quadratic form $h_{s, A}\left[|x|^{\alpha} u\right]$ on $C_{0}^{\infty}\left(\mathbb{R}^{d} \backslash\{0\}\right)$. Moreover, for $n \in \mathbb{N}$ let $W_{n}(x):=\mathcal{C}_{s, d} \min \left\{|x|^{-2(s-\alpha)}, n\right\}$. The boundedness of $W_{n}$, 6.10) and Trotter's product formula show that the diamagnetic inequality is valid for $M_{\beta, A}-W_{n}$. Since $M_{\beta, A}-W_{n} \rightarrow R_{\beta, A}$ in strong resolvent sense again by monotone convergence we find the diamagnetic inequality (6.10) with $M_{\beta, A}$ replaced by $R_{\beta, A}$.

Step 4. Finally, we note that $Q_{\beta, A}=R_{\beta, A}+|x|^{2 \alpha}$ in the sense of quadratic forms. Moreover, $C_{0}^{\infty}\left(\mathbb{R}^{d} \backslash\{0\}\right)$ is a core for both quadratic forms involved. Equation (6.9) now follows from the diamagnetic inequality for $R_{\beta, A}$ by Kato's strong Trotter product formula [ReSi1, Theorem S.21].

6.3. Extension of Theorem 2.1 to magnetic fields. In the case of the nonvanishing magnetic field, the proof of Theorem 2.1 is essentially identical to the one presented in the previous section. Although (5.8) does not necessarily hold with $B_{\beta, A}$ instead of $B_{\beta}$, it does hold if $B_{\beta}$ is replaced by $B_{\beta, A}$ on the left side only! I.e.,

$$
\operatorname{tr}_{\mathfrak{H}_{\beta}} F\left(W^{1 / 2} B_{\beta, A}^{-1} W^{1 / 2}\right) \leq \int_{0}^{\infty} \int_{\mathbb{R}^{d}} \exp \left(-t B_{\beta}\right)(x, x) f(t W(x)) \frac{d x}{|x|^{\beta}} \frac{d t}{t} .
$$

For the proof, one uses the diamagnetic inequality (6.2) before applying Jensen's inequality (cf. Appendix E). This leads to the conclusion that (5.9) also holds with magnetic fields, i.e.,

$$
N\left(-1, H_{s, A}-V\right) \leq K_{p, d, s}^{\prime} \int_{\mathbb{R}^{d}} V(x)_{+}^{p} d x,
$$

with the same ( $A$-independent) constant $K_{p, d, s}^{\prime}$.

For the remainder of the proof, we note that for any $\tau>0$,

$$
N\left(-\tau, H_{s, A}-V\right)=N\left(-1, H_{s, A_{\tau}}-V_{\tau}\right)
$$

where $V_{\tau}(x):=\tau^{-1} V\left(\tau^{-1 / 2 s} x\right)$ and $A_{\tau}(x):=\tau^{-1 / 2 s} A\left(\tau^{-1 / 2 s} x\right)$. The scaling of $A$ does not have any effect, however, since the constant in (6.11) is independent of 
$A$. Therefore (5.11) also holds with $H_{s}$ replaced by $H_{s, A}$, with the same constant $C_{d, s}(\gamma)$.

6.4. The special case $s=1$. As noted in Remark 2.2 the proof of Theorem 2.1 just given also works in the case $s=1$ in dimensions $d \geq 3$. We briefly comment on the necessary modifications.

The local Sobolev-Hardy inequalities for $s=1$ have been proved in BVa. Alternatively, one can obtain them following our proof in Section 3. Using the IMS formula (3.12) one can obtain the global Sobolev-Hardy inequalities (1.4). The rest of the proof goes through without change. To verify the Beurling-Deny criteria, one uses the ground-state representation (4.1) instead of Proposition 4.1. Note also that the weighted diamagnetic inequalities in this section include the case $s=1$.

\section{Appendix A. A constant in the Sobolev inequality (2.6)}

In this appendix we shall derive an explicit bound on the constants $C_{q, 3,1 / 2}$ for the Sobolev-Hardy inequalities (2.6) in the case $d=3$ and $s=1 / 2$, which is of interest for our theorem on stability of matter. Let $3 / 2<q<3$ and $\alpha:=2-3 / q$. For $\lambda>1$, let

$$
\rho(\lambda):=\frac{1-\alpha}{\pi \lambda^{1+\alpha}} \int_{1}^{\infty} \frac{d r}{r^{(1+\alpha) / 2}\left(r-\lambda^{-2}\right)} .
$$

We will show that

$$
C_{q, 3,1 / 2} \leq \frac{\pi^{2}}{3 q^{2}}(1-\alpha)(3 / 4 \pi)^{4 / 3} \inf _{\lambda>1} \frac{\lambda^{2(1-\alpha)}}{\left(\left|\Phi_{1 / 2,3}(\alpha)\right|-\rho(\lambda)\right)_{+}^{2}} .
$$

We remark that in this special case

$$
\left|\Phi_{1 / 2,3}(\alpha)\right|=\frac{2}{\pi}-(1-3 / q) \cot \left(\frac{\pi(1-3 / q)}{2}\right) .
$$

The estimate (A.2) is a consequence of the following two facts. First, we claim that

$$
\limsup _{\delta \rightarrow 0} \limsup _{\varepsilon \rightarrow 0} h_{1 / 2}\left[\psi_{\lambda}^{\varepsilon, \delta}\right] \leq \lambda^{2(1-\alpha)} \frac{\pi^{2}}{3}(1-\alpha),
$$

where $\psi_{\lambda}^{\varepsilon, \delta}(x)=\lambda^{-\alpha} \psi^{\varepsilon, \delta}(x / \lambda)$ and $\psi^{\varepsilon, \delta}$ is defined for $\varepsilon, \delta>0$ by

$$
\psi^{\varepsilon, \delta}(x)= \begin{cases}|x|^{-\alpha} & \text { for }|x| \leq 1 \\ |x|^{-1}\left(1-\varepsilon^{\delta}\left(|x|^{2}-1\right)^{\delta}\right) & \text { for } 1 \leq|x|^{2} \leq 1+1 / \varepsilon \\ 0 & \text { for }|x|^{2} \geq 1+1 / \varepsilon\end{cases}
$$

Note that $\psi^{\varepsilon, \delta}$ does not satisfy the smoothness assumption of Lemma 3.3. but it can be approximated by such functions in $h_{1 / 2}$-norm.

Secondly, we claim that $\rho^{\varepsilon, \delta}$ in (3.10) defined with the function $\psi_{\lambda}=\psi_{\lambda}^{\varepsilon, \delta}$ satisfies

$$
\lim _{\varepsilon \rightarrow 0} \rho^{\varepsilon, \delta}(\lambda)=\rho(\lambda)
$$

uniformly in $\delta>0$ and $\lambda>1$, with $\rho(\lambda)$ as in (A.1). Equation (A.2) then follows from these two facts, proceeding as in the proof of Theorem 2.3. Instead of choosing $\lambda$ such that $\rho(\lambda)=\left|\Phi_{1 / 2,3}(\alpha)\right| / 2$, we now optimize over the choice of $\lambda$.

For the proof of (A.3) we first consider an arbitrary radial function $\psi$ and, with a slight abuse of notation, we write $\psi(x)=\psi(r)$ for $r=|x|$. Using the ground state 
representation in Proposition 4.1, introducing spherical coordinates, and integrating over the angles, we have

$$
h_{1 / 2}[\psi]=8 \int_{0}^{\infty} d r \int_{0}^{\infty} d s \frac{r s}{\left(r^{2}-s^{2}\right)^{2}}|r \psi(r)-s \psi(s)|^{2} .
$$

By changing variables $r^{2} \rightarrow r$ and $s^{2} \rightarrow s$, this yields

$$
h_{1 / 2}[\psi]=2 \int_{0}^{\infty} d r \int_{0}^{\infty} d s \frac{1}{(r-s)^{2}}|\sqrt{r} \psi(\sqrt{r})-\sqrt{s} \psi(\sqrt{s})|^{2} .
$$

Now assume that $\psi=\psi^{\varepsilon, \delta}$ as in A.4. By scaling, if suffices to prove A.3 for $\lambda=1$. We split the integrals in A.6 into several parts. First of all, we have

$$
\begin{array}{rl}
\int_{0}^{1} & d r \int_{0}^{1} d s \frac{1}{(r-s)^{2}}\left(r^{(1-\alpha) / 2}-s^{(1-\alpha) / 2}\right)^{2} \\
& =2 \int_{0}^{1} d s \frac{1}{s^{\alpha}} \int_{0}^{1} d t \frac{1}{(1-t)^{2}}\left(1-t^{(1-\alpha) / 2}\right)^{2} .
\end{array}
$$

This identity can be obtained by noting that the integral on the left is the same as twice the integral over the region $r \leq s$, and then writing $r=s t$ for $0 \leq t \leq 1$. Simple computations then lead to

$$
\begin{aligned}
\int_{0}^{1} d t \frac{1}{(1-t)^{2}}\left(1-t^{(1-\alpha) / 2}\right)^{2} & \\
= & \frac{(1-\alpha)^{2}}{4} \int_{0}^{1} d t \frac{1}{(1-t)^{2}} \int_{t}^{1} d s s^{-(1+\alpha) / 2} \int_{t}^{1} d u u^{-(1+\alpha) / 2} \\
= & \frac{(1-\alpha)^{2}}{2} \int_{0}^{1} d s \int_{s}^{1} d u(s u)^{-(1+\alpha) / 2} \int_{0}^{s} d t \frac{1}{(1-t)^{2}} \\
= & (1-\alpha) \int_{0}^{1} d s \frac{1}{1-s} s^{(1-\alpha) / 2}\left(1-s^{(1-\alpha) / 2}\right)
\end{aligned}
$$

We introduce the function

$$
\eta(\lambda)=\int_{0}^{\infty} d t\left(\frac{e^{-t}}{t}-\frac{e^{-\lambda t}}{1-e^{-t}}\right) .
$$

We note that $\eta(\lambda)=\Gamma^{\prime}(\lambda) / \Gamma(\lambda)$ is the Digamma-function. It is then easy to see that

$$
\int_{0}^{1} d s \frac{1}{1-s} s^{(1-\alpha) / 2}\left(1-s^{(1-\alpha) / 2}\right)=\eta(2-\alpha)-\eta(3 / 2-\alpha / 2) .
$$

Altogether, we conclude that the contribution of $r \leq 1$ and $s \leq 1$ to the integral in (A.6) is given by

$$
4(\eta(2-\alpha)-\eta(3 / 2-\alpha / 2)) .
$$

Similarly, we proceed with the other terms. We have

$$
\begin{aligned}
& \lim _{\varepsilon \rightarrow 0} \int_{0}^{1} d r \int_{1}^{\infty} d s \frac{1}{(r-s)^{2}}\left(r^{(1-\alpha) / 2}-\left[1-\varepsilon^{\delta}(s-1)^{\delta}\right]_{+}\right)^{2} \\
& =\int_{0}^{1} d r \int_{1}^{\infty} d s \frac{1}{(r-s)^{2}}\left(r^{(1-\alpha) / 2}-1\right)^{2}=\int_{0}^{1} d r \frac{1}{1-r}\left(r^{(1-\alpha) / 2}-1\right)^{2}
\end{aligned}
$$

Here we have used dominated convergence, noting that the integrand is bounded from above by the $L^{1}$ function $(r-s)^{-2}\left(2\left(1-r^{(1-\alpha) / 2}\right)^{2}+2 \min \left\{1,(s-1)^{2 \delta}\right\}\right)$ for 
$\varepsilon \leq 1$. The contribution of this term to (A.6) (noting that it appears twice) is thus given by

$$
4(2 \eta(3 / 2-\alpha / 2)-\eta(1)-\eta(2-\alpha)) .
$$

We are left with calculating

$$
\begin{aligned}
& \int_{1}^{1+1 / \varepsilon} d r \int_{1}^{1+1 / \varepsilon} d s \frac{1}{(r-s)^{2}}\left(\varepsilon^{\delta}(r-1)^{\delta}-\varepsilon^{\delta}(s-1)^{\delta}\right)^{2} \\
& =\int_{0}^{1} d r \int_{0}^{1} d s \frac{1}{(r-s)^{2}}\left(r^{\delta}-s^{\delta}\right)^{2}=2(\eta(1+2 \delta)-\eta(1+\delta)) .
\end{aligned}
$$

The last equality follows by proceeding as in (A.7)- The last term to evaluate is

$$
\begin{aligned}
& \int_{1}^{1+1 / \varepsilon} d r \int_{1+1 / \varepsilon}^{\infty} d s \frac{1}{(r-s)^{2}}\left(1-\varepsilon^{\delta}(r-1)^{\delta}\right)^{2} \\
& =\int_{0}^{1} d r \frac{1}{1-r}\left(1-r^{\delta}\right)^{2}=2 \eta(1+\delta)-\eta(1)-\eta(1+2 \delta) .
\end{aligned}
$$

We have thus shown that

$$
\lim _{\varepsilon \rightarrow 0} h_{1 / 2}\left[\psi^{\varepsilon, \delta}\right]=4(\eta(3 / 2-\alpha / 2)+\eta(1+\delta)-2 \eta(1)) .
$$

Using concavity of $\eta$, together with $\eta^{\prime}(1)=\pi^{2} / 6$, yields the estimate

$$
\lim _{\varepsilon \rightarrow 0} h_{1 / 2}\left[\psi^{\varepsilon, \delta}\right] \leq \frac{2 \pi^{2}}{3}\left(\frac{1-\alpha}{2}+\delta\right) .
$$

We proceed similarly for the calculation of $\rho^{\varepsilon, \delta}$. We have

$$
\rho^{\varepsilon, \delta}(\lambda)=\frac{2}{\pi \lambda^{1+\alpha}} \int_{1}^{1+1 / \varepsilon} \frac{r^{(1-\alpha) / 2}}{\left(r-\lambda^{-2}\right)^{2}}\left(1-\frac{1-\varepsilon^{\delta}(r-1)^{\delta}}{r^{(1-\alpha) / 2}}\right) d r .
$$

Equation (A.5) then follows by dominated convergence and integration by parts.

\section{Appendix B. Localization formula in the Magnetic CASE}

In this appendix we establish the analogue of Lemma 3.5 in the general case $A \neq 0$. As explained in Subsection 2.2, this is needed for the proof of Theorem 2.7. First recall that for $s=1$ and $\sum_{j=1}^{n} \chi_{j}^{2} \equiv 1$ one has

$$
\int_{\mathbb{R}^{d}}|(D-A) u|^{2} d x=\sum_{j=0}^{n} \int_{\mathbb{R}^{d}}\left|(D-A)\left(\chi_{j} u\right)\right|^{2} d x-\int_{\mathbb{R}^{d}} \sum_{j=0}^{n}\left|\nabla \chi_{j}\right|^{2}|u|^{2} d x .
$$

In this case the localization error $\sum_{j=0}^{n}\left|\nabla \chi_{j}\right|^{2}$ is local and independent of $A$. The analogue for $s<1$ is

Lemma B.1. Let $d \geq 2,0<s<1$ and $A \in L_{\mathrm{loc}}^{2}\left(\mathbb{R}^{d}\right)$. Then there exists a function $k_{A}$ on $\mathbb{R}^{d} \times \mathbb{R}^{d}$ such that the following holds. If $\chi_{0}, \ldots, \chi_{n}$ are Lipschitz continuous functions on $\mathbb{R}^{d}$ satisfying $\sum_{j=0}^{n} \chi_{j}^{2} \equiv 1$, then one has

$$
\left\||D-A|^{s} u\right\|^{2}=\sum_{j=0}^{n}\left\||D-A|^{s} \chi_{j} u\right\|^{2}-\left(u, L_{A} u\right), \quad u \in \operatorname{dom}|D-A|^{s},
$$


where $L_{A}$ is the bounded operator with integral kernel

$$
L_{A}(x, y):=k_{A}(x, y) \sum_{j=0}^{n}\left(\chi_{j}(x)-\chi_{j}(y)\right)^{2} .
$$

Moreover, for a.e. $x, y \in \mathbb{R}^{d}$

$$
\left|k_{A}(x, y)\right| \leq a_{s, d}|x-y|^{-d-2 s}, \quad \text { and hence } \quad\left|L_{A}(x, y)\right| \leq L(x, y)
$$

with $L$ defined in Lemma 3.5 .

Proof. By the argument of [Si1] we can choose a form core for $|D-A|^{2 s}$ which is invariant under multiplication by Lipschitz continuous functions. It suffices to prove (B.1) only for functions $u$ from such a core.

We write $k_{A}(x, y, t):=\exp \left(-t|D-A|^{2 s}\right)(x, y)$ for the heat kernel and find

$$
\begin{gathered}
\sum_{j=0}^{n}\left(\chi_{j} u,\left(1-\exp \left(-t|D-A|^{2 s}\right)\right) \chi_{j} u\right)=\left(u,\left(1-\exp \left(-t|D-A|^{2 s}\right)\right) u\right) \\
+\frac{1}{2} \sum_{j=0}^{n} \iint k_{A}(x, y, t)\left(\chi_{j}(x)-\chi_{j}(y)\right)^{2} \overline{u(x)} u(y) d x d y .
\end{gathered}
$$

Now we divide by $t$ and note that by our assumption on $u$ the left side converges to $\sum_{j=0}^{n}\left\||D-A|^{s} \chi_{j} u\right\|^{2}$ as $t \rightarrow 0$. Similarly the first term on the right side divided by $t$ converges to $\left\||D-A|^{s} u\right\|^{2}$. Hence the last term divided by $t$ converges to some limit $\left(u, L_{A} u\right)$. The diamagnetic inequality (6.4) yields the bound $\left|k_{A}(x, y, t)\right| \leq$ $\exp \left(-t(-\Delta)^{2 s}\right)(x, y)$. This implies in particular that $L_{A}$ is a bounded operator. Now it is easy to check that $L_{A}$ is an integral operator and that the absolute value of its kernel is bounded pointwise by the one of $L$.

The following helps to clarify the role of the kernel $k_{A}$.

Corollary B.2. Let $u \in \operatorname{dom}|D-A|^{2 s}$ and assume that $\Omega:=\mathbb{R}^{d} \backslash \operatorname{supp} u \neq \emptyset$. Then

$$
\left(|D-A|^{2 s} u\right)(x)=-\int_{\mathbb{R}^{d}} k_{A}(x, y) u(y) d y \quad \text { for } x \in \Omega .
$$

Proof. Let $\varphi \in C_{0}^{\infty}(\Omega)$ and choose $\chi_{0}, \chi_{1}$ such that $\chi_{0} \equiv 1$ on $\operatorname{supp} u, \chi_{1} \equiv 1$ on $\operatorname{supp} \varphi$ and $\chi_{0}^{2}+\chi_{1}^{2} \equiv 1$. By polarization, (B.1) implies $\left(\varphi,|D-A|^{2 s} u\right)=$ $-\left(\varphi, L_{A} u\right)=-\int \overline{\varphi(x)} k_{A}(x, y) u(y) d x d y$, whence the assertion.

\section{Appendix C. Closability of the Quadratic form $b_{\beta, A}$}

This appendix contains some technical details concerning the quadratic form $b_{\beta, A}$ defined in (6.1). In particular, we shall show its closability. Throughout this appendix we assume that $d \geq 2,0<s \leq 1$ and $A \in L_{\mathrm{loc}}^{2}\left(\mathbb{R}^{d}\right)$.

Lemma C.1. The sets $C_{0}^{\infty}\left(\mathbb{R}^{d} \backslash\{0\}\right)$ and $\mathcal{D}:=\left\{w \in \operatorname{dom}(D-A)^{2} \cap L^{\infty}\left(\mathbb{R}^{d}\right)\right.$ : supp $w$ compact in $\left.\mathbb{R}^{d} \backslash\{0\}\right\}$ are form cores for $|D-A|^{2 s}$.

Proof. It suffices to prove the statement for $s=1$. In this case it is proved in Si1] that $C_{0}^{\infty}\left(\mathbb{R}^{d}\right)$ and $\mathcal{D}^{*}:=\left\{w \in \operatorname{dom}(D-A)^{2} \cap L^{\infty}\left(\mathbb{R}^{d}\right): \operatorname{supp} w\right.$ compact $\}$ are form cores for $(D-A)^{2}$. Hence the statement will follow if we can approximate every function in any of these two spaces by functions from the same space vanishing in a neighborhood of the origin. But for functions $u$ from $C_{0}^{\infty}\left(\mathbb{R}^{d}\right)$ or $\mathcal{D}^{*}$ both functions 
$D u$ and $A u$ are square-integrable. This reduces the lemma to the case $A=0$ where it is well-known.

Now let $d-2 s<\beta<d$ and recall that the quadratic form $q_{\beta, A}$ was defined in (6.8). Note that $C_{0}^{\infty}\left(\mathbb{R}^{d} \backslash\{0\}\right)$ is invariant under the unitary transformation in (6.7). Therefore, closability of $q_{\beta, A}$ and $b_{\beta, A}$ on $C_{0}^{\infty}\left(\mathbb{R}^{d} \backslash\{0\}\right)$ are equivalent.

Lemma C.2. The quadratic form $q_{\beta, A}$, defined in (6.8), is closable on $C_{0}^{\infty}\left(\mathbb{R}^{d} \backslash\right.$ $\{0\})$.

Proof. It suffices to show closability of the form $r_{\beta, A}[u]:=h_{s, A}\left[|x|^{\alpha} u\right]$ on $C_{0}^{\infty}\left(\mathbb{R}^{d} \backslash\right.$ $\{0\})$ for $0<\alpha=(\beta+2 s-d) / 2<s$.

Let $\mathcal{D}$ be as in Lemma C.1. We shall show that the quadratic form $r_{\beta, A}$ on $\mathcal{D}$ is closable and that $C_{0}^{\infty}\left(\mathbb{R}^{d} \backslash\{0\}\right)$ is dense with respect to $\left(r_{\beta, A}[w]+\|w\|^{2}\right)^{1 / 2}$ in the closure of $\mathcal{D}$ with respect to this norm. This implies the assertion.

Let $w \in \mathcal{D}$. Since $|x|^{\alpha}$ is smooth on $\operatorname{supp} w$ and $\operatorname{dom}(D-A)^{2}$ is invariant under multiplication by smooth functions we have $|x|^{\alpha} w \in \operatorname{dom}(D-A)^{2}$ and hence $|x|^{\alpha} w \in \operatorname{dom}|D-A|^{2 s}$. Since $|x|^{\alpha} w$ has compact support it follows from Lemma B.1 and Corollary B.2 that $\left|\left(|D-A|^{2 s}|\cdot|^{\alpha} w\right)(x)\right| \leq C(w)|x|^{-d-2 s}$ for all large $|x|$. In particular, $|x|^{\alpha}|D-A|^{2 s}|x|^{\alpha} w \in L^{2}\left(\mathbb{R}^{d}\right)$. Moreover, $|x|^{-2(s-\alpha)} w \in L^{2}\left(\mathbb{R}^{d}\right)$. It follows that if $u_{n} \in \mathcal{D}$ such that $u_{n} \rightarrow 0$ in $L^{2}\left(\mathbb{R}^{d}\right)$, then the bilinear form associated with $r_{\beta, A}$ satisfies

$$
r_{\beta, A}\left[u_{n}, v\right]=\left(u_{n},|x|^{\alpha}|D-A|^{2 s}|x|^{\alpha} w-\mathcal{C}_{s, d}|x|^{-2(s-\alpha)} w\right) \rightarrow 0
$$

as $n \rightarrow \infty$. By standard arguments, this proves that $r_{\beta, A}$ is closable on $\mathcal{D}$.

In order to show the density of $C_{0}^{\infty}\left(\mathbb{R}^{d} \backslash\{0\}\right)$ we again let $w \in \mathcal{D}$. Since $|x|^{\alpha} w \in$ $\operatorname{dom}|D-A|^{s}$, Lemma C.1 yields a sequence $u_{n} \in C_{0}^{\infty}\left(\mathbb{R}^{d} \backslash\{0\}\right)$ such that $\| \mid D-$ $\left.A\right|^{s}\left(u_{n}-|x|^{\alpha} w\right)\|+\| u_{n}-|x|^{\alpha} w \| \rightarrow 0$. Hence, if $w_{n}:=|x|^{-\alpha} u_{n}$, then $w_{n} \rightarrow w$ in $L^{2}\left(\mathbb{R}^{d} \backslash \mathcal{B}\right)$ and $0 \leq r_{\alpha, A}\left[w_{n}-w\right] \leq\left\||D-A|^{s}|x|^{\alpha}\left(w_{n}-w\right)\right\|^{2} \rightarrow 0$. Moreover, Hardy's inequality also implies that $\left\||x|^{-s+\alpha}\left(w_{n}-w\right)\right\| \rightarrow 0$ and hence $w_{n} \rightarrow w$ in $L^{2}(\mathcal{B})$. This proves the density of $C_{0}^{\infty}\left(\mathbb{R}^{d} \backslash\{0\}\right)$ in the closure of $\mathcal{D}$ with respect to $\left(r_{\alpha, A}[w]+\|w\|^{2}\right)^{1 / 2}$.

\section{Appendix D. NASh's ARGUMent}

For the sake of completeness we recall here how the Nash inequality (5.4) and the contraction property of $\exp \left(-t B_{\beta}\right)$ imply the heat kernel estimate (5.3). Let $k(t):=\left\|\exp \left(-t B_{\beta}\right) v\right\|_{\mathfrak{H}_{\beta}}^{2}$. Then

$$
\begin{aligned}
k^{\prime}(t) & =-2 b_{\beta}\left[\exp \left(-t B_{\beta}\right) v\right] \\
& \leq-2 C_{q, d, s}^{-1} k(t)^{1+1 / p}\left\|\exp \left(-t B_{\beta}\right) v\right\|_{L^{1}\left(|x|^{-\beta} d x\right)}^{-2 / p} \\
& \leq-2 C_{q, d, s}^{\prime-1} k(t)^{1+1 / p}\|v\|_{L^{1}\left(|x|^{-\beta} d x\right)}^{-2 / p},
\end{aligned}
$$

where we used (5.4) and the contraction property. Hence

$$
\left(k(t)^{-1 / p}\right)^{\prime} \geq 2 p^{-1} C_{q, d, s}^{-1}\|v\|_{L^{1}\left(|x|^{-\beta} d x\right)}^{-2 / p}
$$

and, after integration,

$$
k(t)^{-1 / p} \geq 2 p^{-1} C_{q, d, s}^{-1} t\|v\|_{L^{1}\left(|x|^{-\beta} d x\right)^{-2 / p}} .
$$


This means that

$$
\left\|\exp \left(-t B_{\beta}\right) v\right\|_{\mathfrak{H}_{\beta}}^{2} \leq\left(p C_{q, d, s}^{\prime} / 2\right)^{p} t^{-p}\|v\|_{L^{1}\left(|x|^{-\beta} d x\right)}^{2} .
$$

By duality, noting that $B_{\beta}$ is self-adjoint in $L^{2}\left(|x|^{-\beta} d x\right)$, this also implies

$$
\left\|\exp \left(-t B_{\beta}\right) v\right\|_{L^{\infty}\left(|x|^{-\beta} d x\right)}^{2} \leq\left(p C_{q, d, s}^{\prime} / 2\right)^{p} t^{-p}\|v\|_{\mathfrak{H}_{\beta}}^{2} .
$$

Finally, by the semi-group property $\exp \left(-t B_{\beta}\right)=\exp \left(-t B_{\beta} / 2\right) \exp \left(-t B_{\beta} / 2\right)$

$$
\left\|\exp \left(-t B_{\beta}\right) v\right\|_{L^{\infty}\left(|x|^{-\beta} d x\right)}^{2} \leq\left(p C_{q, d, s}^{\prime} / 2\right)^{2 p}(t / 2)^{-2 p}\|v\|_{L^{1}\left(|x|^{-\beta} d x\right)}^{2} .
$$

This is exactly the estimate (5.3) with the constant given in Proposition 5.1

\section{Appendix E. A trace estimate}

In this appendix, we sketch the argument leading to Proposition 5.3. We emphasize that we shall ignore several technical details. We assume that $W$ is smooth with compact support in $\mathbb{R}^{d} \backslash\{0\}$, and we put $k(x, y, t):=\exp \left(-t B_{\beta}\right)(x, y)$. We claim that the trace formula

$$
\begin{aligned}
& \operatorname{tr}_{\mathfrak{H}_{\beta}} F\left(W^{1 / 2} B_{\beta}^{-1} W^{1 / 2}\right) \\
& =\int_{0}^{\infty} \frac{d t}{t} \lim _{n \rightarrow \infty} \int_{\mathbb{R}^{d}} \cdots \int_{\mathbb{R}^{d}} \prod_{j=1}^{n} k\left(x_{j}, x_{j-1}, \frac{t}{n}\right) f\left(\frac{t}{n} \sum_{j=1}^{n} W\left(x_{j}\right)\right) \frac{d x_{1}}{\left|x_{1}\right|^{\beta}} \cdots \frac{d x_{n}}{\left|x_{n}\right|^{\beta}}
\end{aligned}
$$

holds true for any non-negative, lower semi-continuous function $f$ vanishing near the origin. Here, $F$ is related to $f$ as in (5.7). In the integral in (E.1) we use the convention that $x_{0}=x_{n}$. Indeed, by an approximation argument it suffices to prove this formula for

$$
F(\lambda)=\lambda /(1+\alpha \lambda), \quad f(\mu)=\mu e^{-\alpha \mu},
$$

where $\alpha>0$ is a constant. Using the resolvent identity and Trotter's product formula, one easily verifies that

$$
\begin{aligned}
F\left(W^{1 / 2} B_{\beta}^{-1} W^{1 / 2}\right) & =W^{1 / 2}\left(B_{\beta}+\alpha W\right)^{-1} W^{1 / 2} \\
& =\int_{0}^{\infty} W^{1 / 2} \exp \left(-t\left(B_{\beta}+\alpha W\right)\right) W^{1 / 2} d t \\
& =\int_{0}^{\infty} \lim _{n \rightarrow \infty} T_{n}(t) d t
\end{aligned}
$$

in this case. Here,

$$
T_{n}(t):=W^{1 / 2}\left(\exp \left(-t B_{\beta} / n\right) \exp (-t \alpha W / n)\right)^{n} W^{1 / 2} .
$$

The latter is an integral operator. We evaluate its trace via integrating its kernel on the diagonal. Then we arrive at

$$
\operatorname{tr}_{\mathfrak{H}_{\beta}} T_{n}(t)=\int \cdots \int \prod_{j=1}^{n} k\left(x_{j}, x_{j-1}, \frac{t}{n}\right) W\left(x_{n}\right) e^{-\frac{\alpha t}{n} \sum_{j} W\left(x_{j}\right)} \frac{d x_{1}}{\left|x_{1}\right|^{\beta}} \cdots \frac{d x_{n}}{\left|x_{n}\right|^{\beta}} .
$$

After symmetrization with respect to the variables this leads to

$$
\operatorname{tr}_{\mathfrak{H}_{\beta}} T_{n}(t)=\frac{1}{t} \int \cdots \int \prod_{j=1}^{n} k\left(x_{j}, x_{j-1}, \frac{t}{n}\right) f\left(\frac{t}{n} \sum_{j} W\left(x_{j}\right)\right) \frac{d x_{1}}{\left|x_{1}\right|^{\beta}} \cdots \frac{d x_{n}}{\left|x_{n}\right|^{\beta}} .
$$


The claimed formula (E.1) follows if one interchanges the trace with the $t$-integration and the $n$-limit.

Now we assume in addition that $f$ is convex. Then Jensen's inequality yields

$$
\begin{aligned}
\int \cdots & \int \prod_{j=1}^{n} k\left(x_{j}, x_{j-1}, \frac{t}{n}\right) f\left(\frac{t}{n} \sum_{j} W\left(x_{j}\right)\right) \frac{d x_{1}}{\left|x_{1}\right|^{\beta}} \cdots \frac{d x_{n}}{\left|x_{n}\right|^{\beta}} \\
& \leq \int \cdots \int \prod_{j=1}^{n} k\left(x_{j}, x_{j-1}, \frac{t}{n}\right) \frac{1}{n} \sum_{j} f\left(t W\left(x_{j}\right)\right) \frac{d x_{1}}{\left|x_{1}\right|^{\beta}} \cdots \frac{d x_{n}}{\left|x_{n}\right|^{\beta}} \\
& =\int \cdots \int \prod_{j=1}^{n} k\left(x_{j}, x_{j-1}, \frac{t}{n}\right) f\left(t W\left(x_{1}\right)\right) \frac{d x_{1}}{\left|x_{1}\right|^{\beta}} \cdots \frac{d x_{n}}{\left|x_{n}\right|^{\beta}} .
\end{aligned}
$$

(Equation (E.1) also holds in the magnetic case discussed in Section 6. Before applying Jensen's inequality, one first has to use the diamagnetic inequality (6.2) to eliminate the magnetic field in the kernel $k$, however.) Finally, we use the semigroup property to integrate with respect to the variables $x_{2}, \ldots, x_{n}$. We find that the latter integral is equal to

$$
\int_{\mathbb{R}^{d}} k(x, x, t) f(t W(x)) \frac{d x}{|x|^{\beta}} .
$$

Plugging this into (E.1) leads to the estimate (5.8).

Details concerning the justification of the above manipulations can be found in RoSo (see Theorem 2.4 there).

\section{ACKNOWLEDGEMENTS}

We thank Heinz Siedentop for suggesting that we study inequalities of this type, and we thank him, Ari Laptev and Jan Philip Solovej for helpful discussions. We also thank Renming Song for valuable comments on a previous version of this manuscript. This work was partially supported by the Swedish Foundation for International Cooperation in Research and Higher Education (STINT) (R.F.), by U.S. National Science Foundation grants PHY 0139984 (E.L.) and PHY 0353181 (R.S.), and by an A.P. Sloan Fellowship (R.S.).

\section{REFERENCES}

[AbSt] M. Abramowitz, I. A. Stegun, Handbook of mathematical functions with formulas, graphs, and mathematical tables. Reprint of the 1972 edition. Dover Publications, New York, 1992. MR1225604 (94b:00012)

[Be1] W. Beckner, Pitt's inequality and the uncertainty principle, Proc. Amer. Math. Soc. 123 (1995), 1897-1905. MR.1254832 (95g:42021)

[Be2] W. Beckner, Pitt's inequality with sharp error estimates, preprint arXiv:math/0701939.

[BL] H. Brezis, E. H. Lieb, Sobolev inequalities with remainder terms. J. Funct. Anal. 62 (1985), no. 1, 73-86. MR0790771 (86i:46033)

[BVa] H. Brezis, J.-L. Vázquez, Blow-up solutions of some nonlinear elliptic problems. Rev. Mat. Univ. Comp. Madrid 10 (1997), 443-469. MR.1605678 (99a:35081)

[Dau] I. Daubechies, An uncertainty principle fermions with a generalized kinetic energy. Comm. Math. Phys. 90 (1983), 511-520. MR0719431 (85j:81008)

[D] E. B. Davies, Heat kernels and spectral theory. Cambridge Tracts in Mathematics 92, Cambridge University Press, Cambridge, 1990. MR1103113 (92a:35035)

[Do] W.F. Donoghue, Monotone matrix functions and analytic continuation. Springer, New York-Heidelberg, 1974. MR0486556 (58:6279) 
[EkFr] T. Ekholm, R. L. Frank, On Lieb-Thirring inequalities for Schrödinger operators with virtual level. Comm. Math. Phys. 264 (2006), no. 3, 725-740. MR2217288 $(2006 \mathrm{~m}: 81101)$

[FLS] R.L. Frank, E.H. Lieb, R. Seiringer, Stability of relativistic matter with magnetic fields for nuclear charges up to the critical value, Commun. Math. Phys. 275 (2007) no. 2, 479-489.

[He] I. W. Herbst, Spectral theory of the operator $\left(p^{2}+m^{2}\right)^{1 / 2}-Z e^{2} / r$. Comm. Math. Phys. 53 (1977), no. 3, 285-294. MR0436854 (55:9790)

[L1] E. H. Lieb, The number of bound states of one-body Schroedinger operators and the Weyl problem. Proc. Sympos. Pure Math., XXXVI, 241-252. Amer. Math. Soc., Providence, R.I., 1980. MR0573436 (82i:35134)

[L2] E. H. Lieb, Sharp Constants in the Hardy-Littlewood-Sobolev and Related Inequalities, Annals of Math. 118 (1983), 349-374. MR0717827 (86i:42010)

[L3] E. H. Lieb, The stability of matter: from atoms to stars. Bull. Amer. Math. Soc. (N.S.) 22 (1990), no. 1, 1-49. MR 1014510 (91f:81002)

[L4] E. H. Lieb, The Stability of Matter and Quantum Electrodynamics, Proceedings of the Heisenberg symposium, Munich, Dec. 2001, Fundamental Physics-Heisenberg and Beyond, G. Buschhorn and J. Wess, eds., pp. 53-68, Springer (2004). A modified version appears in the Milan Journal of Mathematics 71, 199-217 (2003). MR2091506. MR.2120921 (2005k:81363)

[LLo] E. H. Lieb, M. Loss, Analysis. Second edition. Graduate Studies in Mathematics 14, American Mathematical Society, Providence, RI, 2001. MR1817225 (2001i:00001)

[LTh] E. H. Lieb, W. Thirring, Inequalities for the moments of the eigenvalues of the Schrödinger Hamiltonian and their relation to Sobolev inequalities. Studies in Mathematical Physics, 269-303. Princeton University Press, Princeton, NJ, 1976.

[LY] E. H. Lieb, H.-T. Yau, The stability and instability of relativistic matter. Comm. Math. Phys. 118 (1988), no. 2, 177-213. MR0956165 (90c:81251)

[ReSi1] M. Reed, B. Simon, Methods of modern mathematical physics. I. Functional Analysis (Revised and enlarged edition). Academic Press, New York-London, 1980. MR0751959 (85e:46002)

[ReSi2] M. Reed, B. Simon, Methods of modern mathematical physics. IV. Analysis of operators. Academic Press, New York-London, 1978. MR 0493421 (58:12429c)

[RoSo] G. Rozenblyum, M. Solomyak, The Cwikel-Lieb-Rozenblyum estimator for generators of positive semigroups and semigroups dominated by positive semigroups. St. Petersburg Math. J. 9 (1998), no. 6, 1195-1211. MR1610184 (99c:47059)

[Si1] B. Simon, Maximal and minimal Schrödinger forms. J. Operator Theory 1 (1979), no. 1, 37-47. MR0526289 (81m:35104)

[Si2] B. Simon, Functional integration and quantum physics. Second edition. AMS Chelsea Publishing, Providence, RI, 2005. MR2105995 (2005f:81003)

[Ya] D. Yafaev, Sharp constants in the Hardy-Rellich inequalities. J. Funct. Anal. 168 (1999), no. 1, 121-144. MR1717839(2001e:26027)

Department of Mathematics, Royal Institute of Technology, 10044 Stockholm, SweDEN

E-mail address: rupert@math.kth.se

Current address: Department of Mathematics, Princeton University, Princeton, New Jersey 08544

E-mail address: rlfrank@math.princeton.edu

Departments of Mathematics and Physics, Princeton University, P. O. Box 708, Princeton, New Jersey 08544

E-mail address: lieb@princeton.edu

Department of Physics, Princeton University, P. O. Box 708, Princeton, New Jersey 08544

E-mail address: rseiring@princeton.edu 